\title{
Long-lived light neutralinos at Belle II
}

\author{
Sourav Dey, ${ }^{a}$ Claudio O. Dib, ${ }^{b}$ Juan Carlos Helo, ${ }^{c}$ Minakshi Nayak, ${ }^{a}$ Nicolás A. Neill, ${ }^{d}$ \\ Abner Soffer ${ }^{a}$ and Zeren Simon Wang ${ }^{e, f}$ \\ ${ }^{a}$ School of Physics and Astronomy, Tel Aviv University, \\ Tel Aviv 69978, Israel \\ ${ }^{b}$ Departmento de Física and CCTVal, Universidad Técnica Federico Santa María, \\ Valparaiso 2340000, Chile \\ ${ }^{c}$ Departamento de Física, Facultad de Ciencias, Universidad de La Serena, \\ Avenida Cisternas 1200, La Serena, Chile \\ ${ }^{d}$ Instituto de Alta Investigación, Universidad de Tarapacá, \\ Casilla 7D, Arica, Chile \\ ${ }^{e}$ Department of Physics, National Tsing Hua University, \\ Hsinchu 300, Taiwan \\ ${ }^{f}$ Asia Pacific Center for Theoretical Physics (APCTP) - Headquarters San 31, \\ Hyoja-dong, Nam-gu, Pohang 790-784, Korea \\ E-mail: souravdey@tauex.tau.ac.il, claudio.dib@usm.cl, \\ jchelo@userena.cl, minakshi@tauex.tau.ac.il, nicolas.neill@gmail.com, \\ asoffer@tau.ac.il, wzs@mx.nthu.edu.tw
}

ABSTRACT: We consider light neutralinos of mass about $1 \mathrm{GeV}$, produced from $\tau$ lepton rare decays at Belle II, in the context of R-parity-violating (RPV) supersymmetry. With large and clean samples of $\tau$ leptons produced at the Belle II experiment, excellent sensitivity to such light neutralinos with the exotic signatures of displaced vertices is expected. We focus on two benchmark scenarios of single RPV operators, $\lambda_{311}^{\prime} L_{3} Q_{1} \bar{D}_{1}$ and $\lambda_{312}^{\prime} L_{3} Q_{1} \bar{D}_{2}$, which induce both the production and decay of the lightest neutralino. For the reconstruction of a displaced vertex, we require at least two charged pions in the final states. We perform Monte-Carlo simulations for both signal and background events, and find that Belle II can explore regions in the parameter space competitive with other probes. In particular, for the $\lambda_{311}^{\prime}$ scenario, it can put limits up to two orders of magnitude stronger than the current bounds.

Keywords: Supersymmetry Phenomenology

ARXIV EPRINT: 2012.00438 


\section{Contents}

1 Introduction 1

$2 \quad$ Model basics and possible displaced-vertex signatures 3

3 Neutralino production and decay 5

$\begin{array}{lll}4 & \text { Event selection and background estimate } & 6\end{array}$

5 Sensitivity estimation method 11

6 Numerical results 13

$\begin{array}{lll}7 & \text { Conclusions } & 15\end{array}$

A Meson decay constants and branching fractions 16

\section{Introduction}

Supersymmetry (SUSY) [1, 2] remains one of the fore-runners for physics beyond the Standard Model (BSM). In particular, by predicting superpartners for the standard model (SM) particles, whose contributions to the Higgs boson self-energy cancel those of the SM particles, it solves the hierarchy problem $[3,4]$ in an elegant manner. In order to preserve technical naturalness, SUSY predicts such new heavy particles with masses not much higher than the TeV scale. SUSY searches at the Large Hadron Collider (LHC) at CERN in Geneva, Switzerland, have mostly focused on such heavy particles, which are expected to decay promptly into SM particles with large transverse momentum. Furthermore, in the minimal supersymmetric standard model (MSSM), a $Z_{2}$ symmetry called R-parity is usually assumed in order to avoid proton decay. R-parity conservation (RPC) implies that the lightest supersymmetric particle (LSP) is stable. If the LSP is neutral under quantum chromodynamics (QCD) and quantum electrodynamics (QED), its production in squarks and sleptons decays will lead to large transverse missing energy at LHC. However, in SUSY with R-parity-violation (RPV) (see refs. [5-7] for reviews), one can still circumvent the phenomenological issue of proton decay by imposing a different discrete symmetry e.g., the baryon triality $B_{3}$ symmetry [8,9]. Such RPV-SUSY models are equally legitimate and in fact offer a rich phenomenology at colliders. In particular, with RPV, the LSP is no longer stable and can decay into SM particles.

In either the RPC or RPV scenarios, the LHC has so far not discovered SUSY particles of any kind, but only placed $\mathrm{TeV}$-scale lower bounds on the masses of the predicted squarks and gluinos [10-14]. This has led to increased interest in other BSM signatures. 
One class of signatures of interest involves long-lived particles (LLPs), which are generally defined as particles that travel a macroscopic distance before decaying. In fact, even in the SM, such LLPs are common, e.g., the pion, kaon, muon, and neutron. Hence, it is perhaps no surprise that LLPs are predicted in a large number of classes of BSM models. Well studied examples include gauge-mediated, RPV, and split SUSY models, neutral-naturalness models, and portal physics that connects the SM and a "dark sector", where the scalar/fermion/pseudoscalar/vector portal predicts a dark scalar/heavy neutral lepton/axion-like particle/dark photon. For recent reviews of LLP searches, see refs. [15-17].

In this work, we consider a long-lived, light neutralino LSP within the RPV-SUSY. Although there are increasingly stronger lower limits on the squark and gluino masses, this is not the case for the lightest neutralino. In fact, if the GUT (grand-unified theory) relation $M_{1}=\frac{5}{3} \tan ^{2} \theta_{W} M_{2}$ between the Bino and Wino masses is lifted and the dark matter constraint is dropped, the neutralino can be as light as in the $\mathrm{GeV}$ scale or even massless [18-26]. Such light neutralinos are also consistent with both astrophysical and cosmological constraints [27-33]. Note that in order to avoid overclosing the Universe [3437], light neutralinos should decay, e.g., in the context of the RPV-SUSY. Moreover, for a range of values of the RPV couplings, $\mathrm{GeV}$-scale neutralinos are long-lived and have a significant probability of decaying inside a detector. Such decays lead to a displaced-vertex (DV) signature at colliders, which is the signature we explore here.

The GeV-scale neutralinos are necessarily Bino-like in order to avoid the present bounds $[25,26]$. In the literature, two main production mechanisms have been discussed and studied for the LHC [38], future lepton colliders [39, 40], and a list of proposed extended programs at the LHC [41-44]: 1) pair production in $Z$-boson rare decays via the small Higgsino component, and 2) single production in rare decays of charm and bottom mesons via an RPV coupling. In this work, for the first time, we propose to consider light neutralinos produced in $\tau$ lepton decays.

$\tau$ leptons are copiously produced at colliders, including the LHC, $B$-factories, and $\tau$-charm threshold colliders [45]. Here we focus on the ongoing Belle II experiment in Japan $[46,47]$ at the intensity-frontier, where electron and positron beams are colliding at a center-of-mass energy $\sqrt{s}=10.58 \mathrm{GeV}$. The projected total integrated luminosity of Belle II, to be collected in the next few years, is $50 \mathrm{ab}^{-1}$, roughly 50 times that of the previous Belle experiment. This corresponds to a sample of $4.6 \times 10^{10} e^{+} e^{-} \rightarrow \tau^{+} \tau^{-}$ events. The resulting events are easily identifiable via the back-to-back production of the $\tau$ pairs and the large missing momentum. These properties make Belle II one of the best facilities for the study of rare $\tau$ decays.

The LLP search potential of Belle II and the previous-generation $B$-factories, BABAR [48] and Belle [49], for a variety of models has been investigated extensively (see for instance refs. [50-57]). In particular, ref. [55] considered heavy neutral leptons as the LLPs produced from $\tau$ decays at Belle II, using as a benchmark model a heavy neutral lepton that mixes predominantly with the third-generation active neutrino $\nu_{\tau}$.

This paper is structured as follows. Section 2 introduces the RPV-SUSY model considered in this work, followed by section 3 in which we present the analytic formulas of $\tau$ 
and neutralino decays. In section 4 we explain the signature definition and background estimate, followed by section 5 where we elaborate on the sensitivity estimate procedure via a Monte-Carlo (MC) simulation. We present the numeric results in section 6. Finally, we summarize the work and offer an outlook in section 7 .

\section{Model basics and possible displaced-vertex signatures}

We consider a model where the MSSM is appended with R-parity violation (RPV-MSSM). With the R-parity violation, the usual MSSM superpotential is extended with the following terms:

$$
W_{\mathrm{RPV}}=\epsilon_{i} L_{i} \cdot H_{u}+\frac{1}{2} \lambda_{i j k} L_{i} \cdot L_{j} \bar{E}_{k}+\lambda_{i j k}^{\prime} L_{i} \cdot Q_{j} \bar{D}_{k}+\frac{1}{2} \lambda_{i j k}^{\prime \prime} \bar{U}_{i} \bar{D}_{j} \bar{D}_{k},
$$

where the indices $i, j, k$ refer to any of the three fermion generations. The first three sets of terms are lepton-number-violating, and the last set violates baryon number. Allowing all the terms would lead to a too large proton decay rate, unless the couplings were extremely small. As mentioned above, imposing certain discrete symmetries, such as the baryon triality, can remove, e.g., the baryon-number-violating terms, thus forbidding proton decay. For simplicity, we assume that only certain $\lambda_{i j k}^{\prime} L_{i} Q_{j} \bar{D}_{k}$ operators are non-vanishing. More concretely, we focus on the operators $\lambda_{311}^{\prime} L_{3} Q_{1} \bar{D}_{1}$ and $\lambda_{312}^{\prime} L_{3} Q_{1} \bar{D}_{2}$, which are relevant for $\tau$ decay.

In the RPV-MSSM, while the decay of the lightest neutralino can only proceed via one or more RPV couplings, its production can be mediated via different mechanisms. First, despite the Bino-like nature of such light neutralinos, the small Higgsino component allows for coupling with a $Z$-boson. If $\tilde{\chi}_{1}^{0}$ is lighter than $M_{Z} / 2$, the decay $Z \rightarrow \tilde{\chi}_{1}^{0} \tilde{\chi}_{1}^{0}$ may ensue. This scenario has been studied in the context of both LHC experiments and future $Z$ factories. Second, the $L Q \bar{D}$ operators can lead to the decay of charm and bottom mesons into the lightest neutralino. This was proposed for the first time in ref. [38]. Providing the analytic expressions of the decay widths of the mesons and light neutralinos, the authors studied the sensitivity of ATLAS and the fixed-target experiment SHiP [58]. It is worth mentioning that the authors of ref. [38] estimated the transition form factors for both the second and third generations of quarks using the heavy quark formulation. While they cannot rigorously apply such a method to purely first-generation flavors, they state that it is reasonable to follow the same approach and to assume that the tensor and vector decay constants satisfy $f_{T}=f_{V}$ for first-generation mesons as well, within the expected level of precision determined by other uncertainties. In our benchmark scenarios, which are dominated by pseudoscalar mesons, we use the same assumption because the impact of the uncertainty in $f_{T}$ is even smaller.

In this paper we consider the case of one single RPV operator, either $\lambda_{311}^{\prime} L_{3} Q_{1} \bar{D}_{1}$ only or $\lambda_{312}^{\prime} L_{3} Q_{1} \bar{D}_{2}$ only, mediating both the production and decay of a light neutralino. As shown in figure 1 , the $\tau^{-}$decay produces a charged pseudoscalar (vector) meson $M_{1}$ $\left(M_{1}^{*}\right)$, with quark content $d \bar{u}$ (namely, $\pi^{-}, \rho^{-}$) in the case of $\lambda_{311}^{\prime} \neq 0$, or $s \bar{u}\left(K^{-}, K^{*-}\right.$ ) in the case of $\lambda_{312}^{\prime} \neq 0$. The same $L Q \bar{D}$ operator also gives rise to the neutralino decay. 

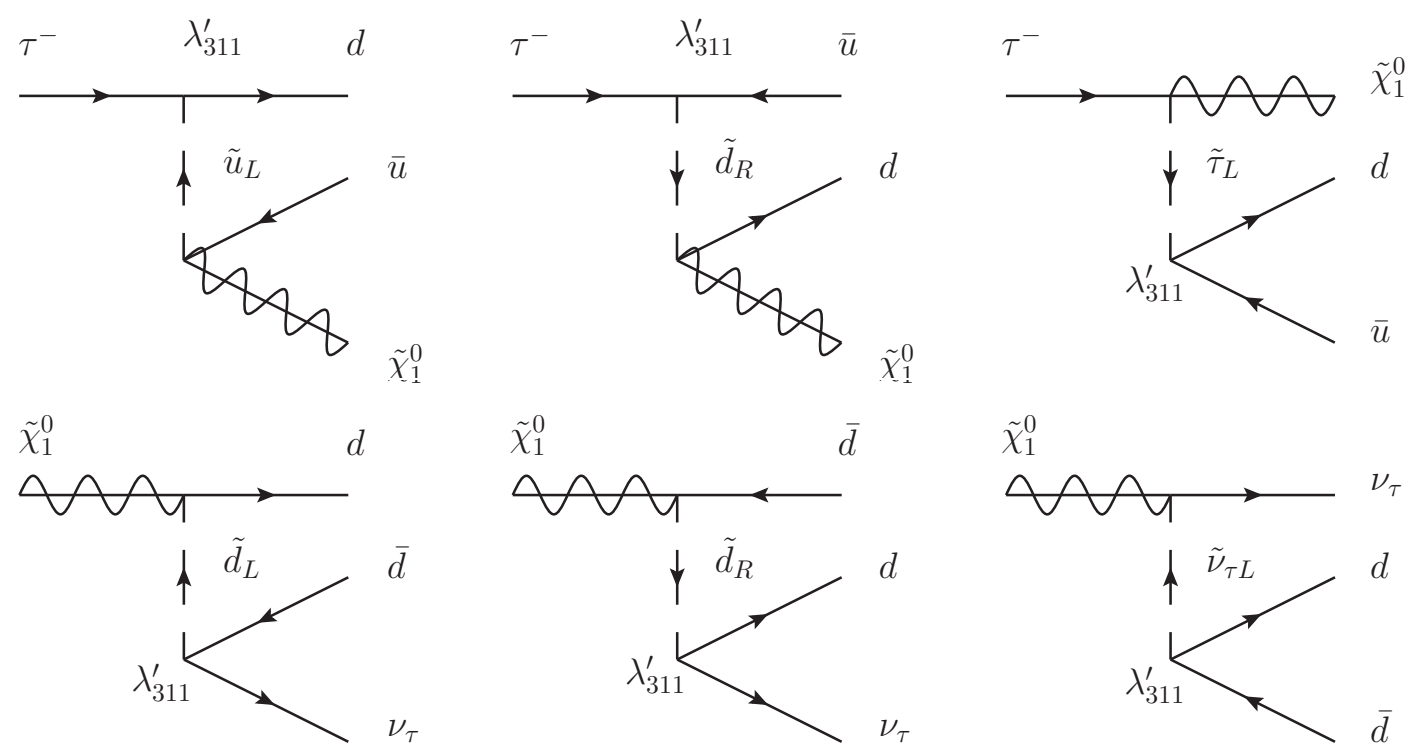

Figure 1. The parton-level Feynman diagrams for both $\tau$ and $\tilde{\chi}_{1}^{0}$ decays via the coupling $\lambda_{311}^{\prime}$. The diagrams for the coupling $\lambda_{312}^{\prime}$ can be easily obtained by changing a $d$ (s)quark to an $s$ (s)quark.

Since the neutralino is produced from $\tau$ decays, its mass satisfies $m_{\tilde{\chi}_{1}^{0}}<m_{\tau}$. As a result, it can only decay into $\nu_{\tau}+M_{2}^{(*)}$, where $M_{2}^{(*)}$ is a pseudoscalar (vector) neutral meson with quark content $d \bar{d}$ (forming either a $\pi^{0}, \rho^{0}, \eta, \eta^{\prime}$, or $\omega$ ) for the $\lambda_{311}^{\prime}$ coupling, or $d \bar{s}$ (forming a $K^{0}, K^{* 0}$ ) for the $\lambda_{312}^{\prime}$ coupling (see figure 1). Except for the $\pi^{0}$ and $K^{0}$, these mesons decay promptly into charged hadrons, enabling the identification of the neutralino displaced decay position. When $M_{2}$ is a $K^{0}$ that decays as $K_{S} \rightarrow \pi^{+} \pi^{-}$, the DV does not identify the decay position of the neutralino, because of the long lifetime of the $K_{S}$ $\left(c \tau_{K_{S}} \approx 2.7 \mathrm{~cm}\right)$. Nonetheless, this signature can be used to detect a signal if the neutralino flight distance is significantly larger than $\left(p_{K_{S}} / m_{K_{S}}\right) c \tau_{K_{S}}$ and the momentum vector of the $K_{S}$ does not point back to the $\tau$ decay position. Owing to the relatively short lifetime of the $\tau$, its decay position can be safely taken to be the interaction point (IP) of the collider beams.

The current bound on $\lambda_{31 k}^{\prime}$ is calculated in ref. [59] for the case in which the virtual sfermion is a squark with mass $m_{\tilde{q}_{R}} \gtrsim 1 \mathrm{TeV}$ :

$$
\left|\lambda_{31 k}^{\prime}\right|<0.20 \frac{m_{\tilde{d}_{R k}}}{1 \mathrm{TeV}}+0.046 .
$$

This limit was obtained by recasting a $p p \rightarrow W^{\prime} \rightarrow \tau \nu_{\tau}$ search for high-mass resonances decaying to a tau lepton and a neutrino, performed at ATLAS with $36.1 \mathrm{fb}^{-1}$ of data [60]. A similar search was performed by CMS [61]. The expected bound at the HL-LHC with $3 \mathrm{ab}^{-1}$ is also given in Ref. [59]. Since it is only slightly tighter than the present bound of eq. (2.2), we use only the present bound for comparison to our results below.

An additional bound comes from the current uncertainties in the measured branching fractions for $\tau \rightarrow P \nu_{\tau}(P=\pi, K)[62]$ and the uncertainty in their theoretical predic- 
tions [63],

$$
\begin{aligned}
\mathcal{B}\left(\tau \rightarrow \pi \nu_{\tau}\right)_{\mathrm{EXP}} & =(10.82 \pm 0.05) \%, \quad \mathcal{B}\left(\tau \rightarrow K \nu_{\tau}\right)_{\mathrm{EXP}}=(0.696 \pm 0.01) \% \\
\mathcal{B}\left(\tau \rightarrow \pi \nu_{\tau}\right)_{\mathrm{SM}} & =(10.90 \pm 0.027) \%, \quad \mathcal{B}\left(\tau \rightarrow K \nu_{\tau}\right)_{\mathrm{SM}}=(0.722 \pm 0.004) \%
\end{aligned}
$$

where for the SM values, quoted as a function of the $\tau$ lifetime in ref. [63], we use the current PDG average value $\tau_{\tau}=(290.3 \pm 0.5)$ fs. From the combined experimental and theoretical uncertainties in $\mathcal{B}\left(\tau \rightarrow P \nu_{\tau}\right)$,

$$
\sigma_{\mathcal{B}\left(\tau \rightarrow \pi \nu_{\tau}\right)}=0.057 \times 10^{-2}, \quad \sigma_{\mathcal{B}\left(\tau \rightarrow K \nu_{\tau}\right)}=0.011 \times 10^{-2},
$$

we extract the following $95 \%$ confidence level bounds on $\mathcal{B}\left(\tau \rightarrow P \tilde{\chi}_{1}^{0}\right)$

$$
\mathcal{B}\left(\tau \rightarrow P \tilde{\chi}_{1}^{0}\right) \lesssim 2 \sigma_{\mathcal{B}\left(\tau \rightarrow P \nu_{\tau}\right)}
$$

This limit is relevant only for large neutralino lifetimes. For short lifetimes, the neutralino daughter particles are in principle visible in the detector and might be rejected from the $\tau \rightarrow P \tilde{\chi}_{1}^{0}$ candidate sample, depending on the analysis criteria. Therefore, we show this bound with our results in section 6 only for $c \tau_{\tilde{\chi}_{1}^{0}}>1 \mathrm{~m}$. We ignore the fact that this bound becomes inaccurate when $m_{\tilde{\chi}_{1}^{0}}$ approaches $m_{\tau}$, so that the $\tau \rightarrow P \tilde{\chi}_{1}^{0}$ event selection may reject the resulting soft pseudoscalar.

\section{Neutralino production and decay}

We extract the effective interaction Lagrangian from ref. [38],

$$
\begin{aligned}
\mathcal{L}= & G_{i j k}^{S, \nu}\left(\overline{\widetilde{\chi}^{0}} P_{L} \nu_{i}\right)\left(\overline{d_{k}} P_{L} d_{j}\right)+G_{i j k}^{S, \ell}\left(\overline{\widetilde{\chi}^{0}} P_{L} \ell_{i}\right)\left(\overline{d_{k}} P_{L} u_{j}\right) \\
& +G_{i j k}^{T, \nu}\left(\overline{\widetilde{\chi}^{0}} \sigma^{\mu \nu} \nu_{i}\right)\left(\overline{d_{k}} \sigma^{\rho \sigma} d_{j}\right)\left(g_{\mu \rho} g_{\nu \sigma}-\frac{i \epsilon_{\mu \nu \rho \sigma}}{2}\right) \\
& +G_{i j k}^{T, \ell}\left(\overline{\widetilde{\chi}^{0}} \sigma^{\mu \nu} \ell_{i}\right)\left(\overline{d_{k}} \sigma^{\rho \sigma} u_{j}\right)\left(g_{\mu \rho} g_{\nu \sigma}-\frac{i \epsilon_{\mu \nu \rho \sigma}}{2}\right)+\text { h.c. }
\end{aligned}
$$

where the effective couplings $G_{i j k}^{S, T}$ are proportional to the corresponding $\lambda_{i j k}^{\prime}$ couplings,

$$
\begin{aligned}
G_{i j k}^{S, \ell}=\lambda_{i j k}^{\prime}\left(\frac{1}{2} \frac{g_{\tilde{u}_{L}}}{m_{\tilde{u}_{j L}}^{2}}+\frac{1}{2} \frac{g_{\tilde{d}_{R}}^{*}}{m_{\tilde{d}_{k R}}^{2}}-\frac{g_{\tilde{\ell}_{L}}}{m_{\tilde{\ell}_{i L}}^{2}}\right), & G_{i j k}^{T, \nu}=\lambda_{i j k}^{\prime}\left(\frac{g_{\tilde{d}_{L}}}{4 m_{\tilde{d}_{j L}}^{2}}+\frac{g_{\tilde{d}_{R}}^{*}}{4 m_{\tilde{d}_{k R}}^{2}}\right), \\
G_{i j k}^{S, \nu}=\lambda_{i j k}^{\prime}\left(\frac{g_{\tilde{\nu}_{L}}}{m_{\tilde{\nu}_{i L}}^{2}}-\frac{1}{2} \frac{g_{\tilde{d}_{L}}}{m_{\tilde{d}_{j L}}^{2}}-\frac{1}{2} \frac{g_{\tilde{d}_{R}}^{*}}{m_{\tilde{d}_{k R}}^{2}}\right), & G_{i j k}^{T, \ell}=\lambda_{i j k}^{\prime}\left(\frac{g_{\tilde{u}_{L}}}{4 m_{\tilde{u}_{j L}}^{2}}+\frac{g_{\tilde{d}_{R}}^{*}}{4 m_{\tilde{d}_{k R}}^{2}}\right),
\end{aligned}
$$

and the coupling constants $g_{\tilde{f}}$ are given by the electroweak $\mathrm{SU}(2)$ gauge coupling $g_{2}$ and the electroweak mixing angle $\theta_{W}$ :

$$
g_{\tilde{\ell}_{L}}=g_{\tilde{\nu}_{L}}=\frac{g_{2}}{\sqrt{2}} \tan \theta_{W}, \quad g_{\tilde{u}_{L}}=g_{\tilde{d}_{L}}=-\frac{g_{2}}{3 \sqrt{2}} \tan \theta_{W}, \quad g_{\tilde{d}_{R}}=-\frac{2 g_{2}}{3 \sqrt{2}} \tan \theta_{W} .
$$


From the effective Lagrangian, the neutralino production rate in association with a scalar meson $M_{1}$ or a vector meson $M_{1}^{*}$ is

$$
\begin{aligned}
\Gamma\left(\tau \rightarrow M_{1} \tilde{\chi}_{1}^{0}\right)= & \frac{\lambda^{1 / 2}\left(m_{\tau}^{2}, m_{M_{1}}^{2}, m_{\tilde{\chi}_{1}^{0}}^{2}\right)}{128 \pi m_{\tau}^{3}}\left|G_{3 j k}^{S, \ell}\right|^{2}\left|f_{M_{1}}^{S}\right|^{2}\left(m_{\tau}^{2}-m_{M}^{2}+m_{\tilde{\chi}_{1}^{0}}^{2}\right), \\
\Gamma\left(\tau \rightarrow M_{1}^{*} \tilde{\chi}_{1}^{0}\right)= & \frac{\lambda^{1 / 2}\left(m_{\tau}^{2}, m_{M_{1}^{*}}^{2}, m_{\tilde{\chi}_{1}^{0}}^{2}\right)}{2 \pi m_{\tau}^{3}}\left|G_{3 j k}^{T, \ell}\right|^{2}\left|f_{M_{1}^{*}}^{T}\right|^{2} \\
& \times\left[2\left(m_{\tau}^{2}-m_{\tilde{\chi}_{1}^{0}}^{2}\right)^{2}-m_{M_{1}^{*}}^{2}\left(m_{M_{1}^{*}}^{2}+m_{\tau}^{2}+m_{\tilde{\chi}_{1}^{0}}^{2}\right)\right],
\end{aligned}
$$

where the scalar and tensor decay constants $f_{M_{1}}^{S}, f_{M_{1}^{*}}^{T}$ are given in appendix A. For the decays of the neutralino in our scenario, we reproduce the expressions given in ref. [38],

$$
\begin{aligned}
& \Gamma\left(\tilde{\chi}_{1}^{0} \rightarrow M_{2} \nu_{\tau}\right)=\frac{\lambda^{1 / 2}\left(m_{\tilde{\chi}_{1}^{0}}^{2}, m_{M_{2}}^{2}, 0\right)}{128 \pi m_{\tilde{\chi}_{1}^{0}}^{3}}\left|G_{3 j k}^{S, \nu_{\tau}}\right|^{2}\left|f_{M_{2}}^{S}\right|^{2}\left(m_{\tilde{\chi}_{1}^{0}}^{2}-m_{M_{2}}^{2}\right), \\
& \Gamma\left(\tilde{\chi}_{1}^{0} \rightarrow M_{2}^{*} \nu_{\tau}\right)=\frac{\lambda^{1 / 2}\left(m_{\tilde{\chi}_{1}^{0}}^{2}, m_{M_{2}^{*}}^{2}, 0\right)}{2 \pi m_{\tilde{\chi}_{1}^{0}}^{3}}\left|G_{3 j k}^{T, \nu_{\tau}}\right|^{2}\left|f_{M_{2}^{*}}^{T}\right|^{2}\left[2 m_{\tilde{\chi}_{1}^{0}}^{4}-m_{M_{2}^{*}}^{2}\left(m_{M_{2}^{*}}^{2}+m_{\tilde{\chi}_{1}^{0}}^{2}\right)\right],
\end{aligned}
$$

where $\lambda(x, y, z)=x^{2}+y^{2}+z^{2}-2 x y-2 x z-2 y z$. Note that the charge-conjugate channel for the $\tilde{\chi}_{1}^{0}$ decays is implied. For simplicity, we assume degenerate sfermion masses, henceforth denoted $m_{\tilde{f}}$, enabling a study of the sensitivity in terms of the ratio $\lambda^{\prime} / m_{\tilde{f}}^{2}$.

Table 1 lists the different decay modes that we consider for the cases of non-zero $\lambda_{311}^{\prime}$ or $\lambda_{312}^{\prime}$. In figures 2 and 3 we show the rates for the different neutralino production and decay modes, as well as the decay branching ratios of the neutralino to visible modes. It is evident that neutralino decays into pseudoscalar $M_{2}$ mesons, namely, $\eta$ and $\eta^{\prime}$, are by far dominant, and hence constitute the best search strategies. For this reason, in the case $\lambda_{311}^{\prime} \neq 0$ we do not consider neutralino production associated with the $a_{1}^{-}$meson, i.e. $\tau^{-} \rightarrow \tilde{\chi}_{1}^{0} a_{1}^{-}$. Since $m_{\tau}-m_{a_{1}}<m_{\eta}$, this mode does not contribute to visible DVs in this scenario and hence is irrelevant in this estimate. Also shown in the figures are the neutralino branching fractions into final states with at least two charged pions, which are needed for reconstruction of the DV and its position.

\section{Event selection and background estimate}

The search proposed here is experimentally very similar to that of the heavy neutral lepton (HNL) search proposed in ref. [55]. Therefore, the background suppression and estimation methods, as well as conclusions about the major sources of background, are similar to those of ref. [55]. In particular, the selection of signal events will begin with the typical $e^{+} e^{-} \rightarrow \tau^{+} \tau^{-}$selection criteria. These include a single track that recoils against the rest of the event in the opposite hemisphere, accompanied by large missing energy and transverse momentum [55, 64]. This selection leaves the backgrounds from $e^{+} e^{-} \rightarrow \tau^{+} \tau^{-}$events as the dominant source, efficiently rejecting all other sources of background.

Subsequently, the main LLP requirement involves selection of two identified charged pions that originate from a high-quality vertex that is significantly displaced from the 


\begin{tabular}{|c|c|c|}
\hline \multicolumn{2}{|c|}{$\tau \rightarrow \tilde{\chi}_{1}^{0} M_{1}^{(*)}, \tilde{\chi}_{1}^{0} \rightarrow M_{2}^{(*)} \nu_{\tau}$} \\
\hline$\lambda^{\prime}$ (production and decay) & Scenario 1 & Scenario 2 \\
\hline Mesons in $\tilde{\chi}_{1}^{0}$ production $\left(M_{1}\right)$ & $\lambda_{311}^{\prime}$ & $\lambda_{312}^{\prime}$ \\
\hline Mesons in $\tilde{\chi}_{1}^{0}$ decay $\left(M_{2}\right)$ & $\pi^{ \pm}, \rho^{ \pm}$ & $K^{ \pm} K^{* \pm}$ \\
\hline$M_{2}$ decays with charged & $\pi^{0}, \rho, \omega, \eta, \eta^{\prime}$ & $K^{0}, K^{* 0}$ \\
\hline particles & $\eta \rightarrow \pi^{+} \pi^{-} \gamma, \eta \rightarrow \pi^{+} \pi^{-} \pi^{0}$, & \\
& $\begin{array}{c}\eta^{\prime} \rightarrow \pi^{+} \pi^{-}\left(\eta \rightarrow 3 \pi^{0}\right), \eta^{\prime} \rightarrow \pi^{0} \pi^{0}\left(\eta \rightarrow \pi^{+} \pi^{-} \pi^{0}\right), \\
\eta^{\prime} \rightarrow \pi^{+} \pi^{-}(\eta \rightarrow \gamma \gamma), \eta^{\prime} \rightarrow \pi^{0} \pi^{0}\left(\eta \rightarrow \pi^{+} \pi^{-} \gamma\right) \\
\eta^{\prime} \rightarrow \gamma\left(\omega \rightarrow \pi^{+} \pi^{-} \pi^{0}\right), \eta^{\prime} \rightarrow \gamma\left(\rho \rightarrow \pi^{+} \pi^{-}\right) \\
\end{array}$ & $K^{* 0} \rightarrow \pi^{ \pm} K^{\mp}$ \\
& $\eta^{\prime} \rightarrow \gamma\left(\omega \rightarrow \pi^{+} \pi^{-}\right), \eta^{\prime} \rightarrow \pi^{+} \pi^{-}\left(\eta \rightarrow \pi^{+} \pi^{-} \gamma\right)$ & \\
& $\eta^{\prime} \rightarrow \pi^{+} \pi^{-}\left(\eta \rightarrow \pi^{+} \pi^{-} \pi^{0}\right)$ & \\
\hline
\end{tabular}

Table 1. Benchmark scenarios for neutralinos produced from $\tau$ decays: $\tau \rightarrow \tilde{\chi}_{1}^{0} M_{1}^{(*)}, \tilde{\chi}_{1}^{0} \rightarrow M_{2}^{(*)} \nu_{\tau}$.
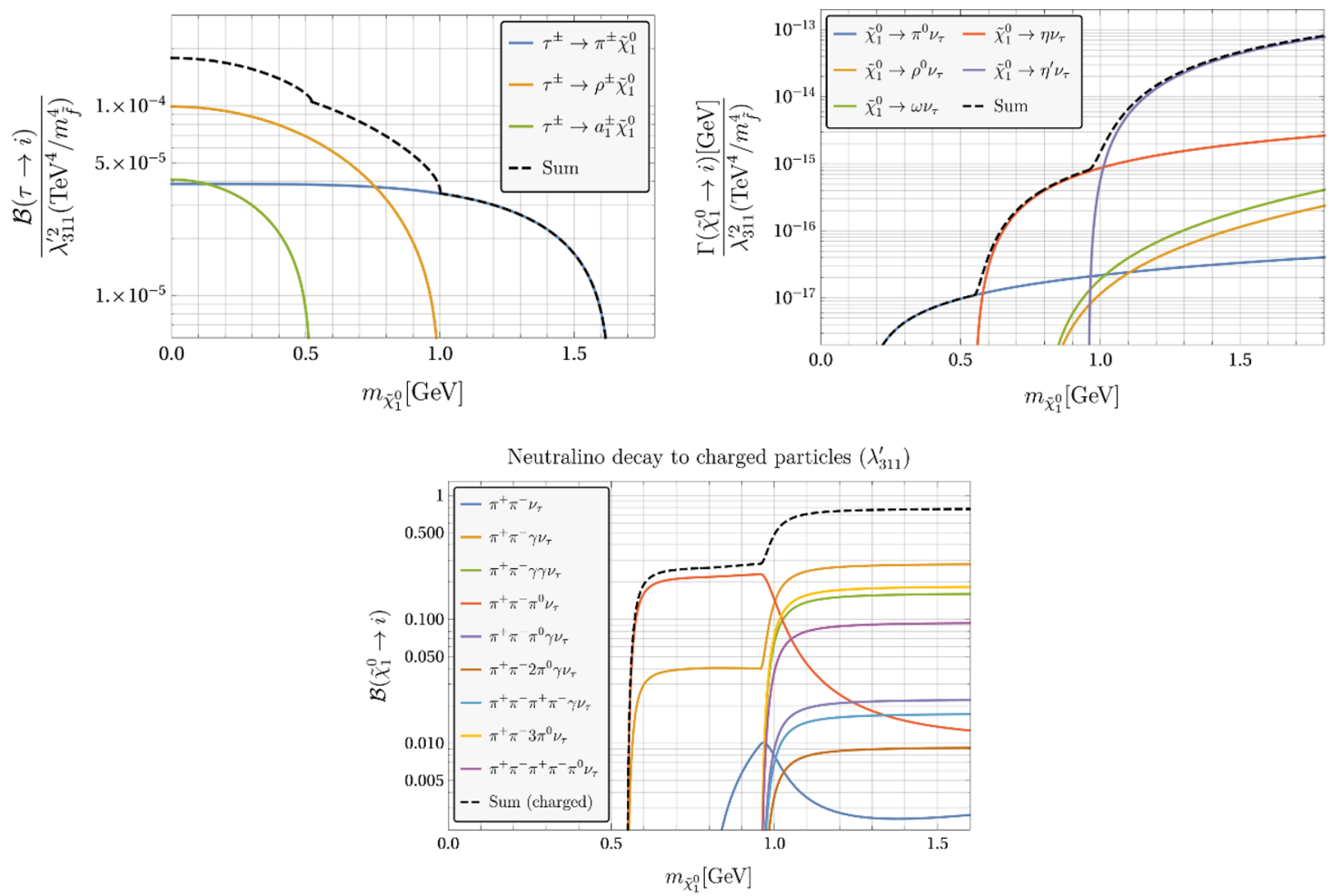

Figure 2. Neutralino production and decay in the $\lambda_{311}^{\prime} \neq 0$ scenario. (Top left) Branching fractions $\mathcal{B}\left(\tau \rightarrow M_{1} \tilde{\chi}_{1}^{0}\right)$ for $\tau$ decays into a meson and a neutralino. (Top right) Decay rates $\Gamma\left(\tilde{\chi}_{1}^{0} \rightarrow M_{2} \nu_{\tau}\right)$ for neutralino decays into a meson and a neutrino. (Bottom) Branching fractions for neutralino decays into final states with charged particles, accounting for the decays of the $M_{2}$ mesons. 

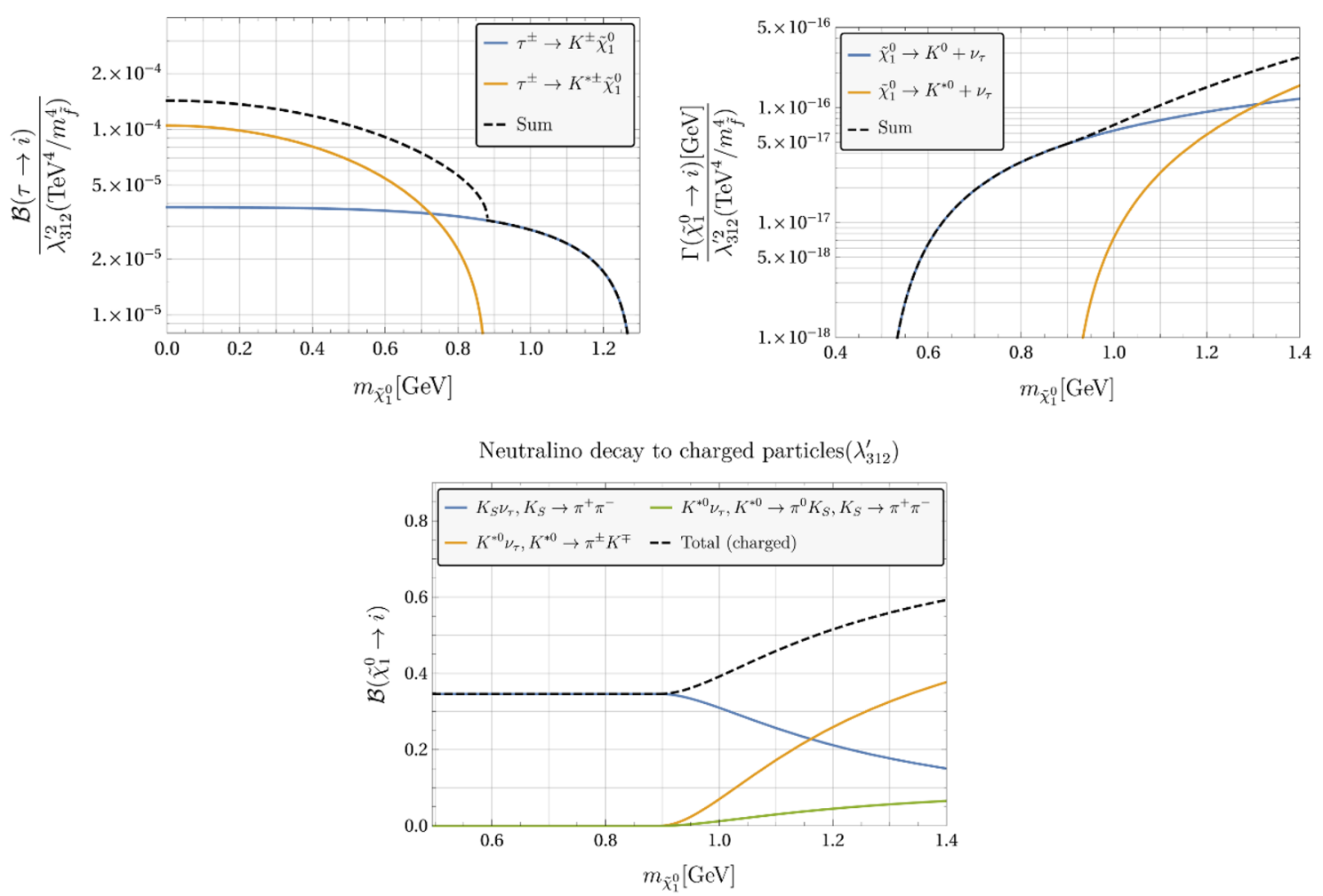

Figure 3. Neutralino production and decay in the $\lambda_{312}^{\prime} \neq 0$ scenario. (Top left) Branching fractions $\mathcal{B}\left(\tau \rightarrow M_{1} \tilde{\chi}_{1}^{0}\right)$ for $\tau$ decays into a meson and a neutralino. (Top right) Decay rates $\Gamma\left(\tilde{\chi}_{1}^{0} \rightarrow M_{2} \nu_{\tau}\right)$ for neutralino decays into a meson and a neutrino. (Bottom) Branching fractions for neutralino decays into final states with charged particles, accounting for the decays of the $M_{2}$ mesons.

IP. Furthermore, a key difference with respect to ref. [55] is that the neutralino decay produces a narrow hadron, namely, a $\eta$ or $\eta^{\prime}$, which is rarely produced in $\tau$ decays [64-67]. Requiring the presence of a $\eta$ or $\eta^{\prime}$ decay that occurs far from the interaction point will be very effective at suppressing the background. In what follows we estimate the background for the signal case $\tau^{-} \rightarrow \pi^{-} \tilde{\chi}_{1}^{0}, \tilde{\chi}_{1}^{0} \rightarrow \eta \nu_{\tau}, \eta \rightarrow \pi^{+} \pi^{-} \pi^{0}$ in detail. We then qualitatively extend our conclusions to the other signal modes.

Naïvely, since the $\eta$ is reconstructed in the final state $\pi^{+} \pi^{-} \pi^{0}$, a conceivable source of background is $\tau^{-} \rightarrow \pi^{-} K_{L} \nu_{\tau}$, followed by the decay of the long-lived $K_{L}$ to $\pi^{+} \pi^{-} \pi^{0}$. The branching fractions of these two decays are $4.2 \times 10^{-3}$ and $12.5 \%$, respectively. The $\pi^{+} \pi^{-} \pi^{0}$ mass resolution is less than $4 \mathrm{MeV}$ at $B$-factories [64]. Therefore, even if one accounts for the resolution degradation in the case of a displaced decay, the likelihood of a $K_{L}$ decay faking an $\eta$ is greatly suppressed, given that the masses of these mesons differ by about $50 \mathrm{MeV}$.

We estimate that the largest source of background will arise from $\tau^{-} \rightarrow \pi^{-} \pi_{p}^{0} K_{L} \nu_{\tau}$, which has a branching fraction of $1.7 \times 10^{-3}$, followed by $K_{L} \rightarrow \pi^{+} \pi^{-} \pi_{d}^{0}$, for which the branching fraction is $12.5 \%$. For clarity, we denote the prompt and displaced $\pi^{0}$ mesons with the subscripts $p$ and $d$, respectively. The photons that are produced in the $\pi_{p}^{0}$ and $\pi_{d}^{0}$ decays are denoted $\gamma_{p}^{i}$ and $\gamma_{d}^{i}$, with $i=1,2$. A fake $\eta \rightarrow \pi^{+} \pi^{-} \pi^{0}$ decay candidate 
can then be reconstructed with the displaced $\pi^{ \pm}$and either the $\pi_{p}^{0}$ or a fake $\pi^{0}$ candidate formed from $\gamma_{p}^{i} \gamma_{d}^{j}$. The two remaining photons would, if detected, indicate that this is not a signal event. However, they may escape via the endcap openings in the calorimeter or otherwise go undetected, typically since they are too soft to be clearly identified above photon background that originates from the collider itself.

To estimate the level of this background, we use the EvtGen [68] Monte-Carlo generator to produce $e^{+} e^{-} \rightarrow \tau^{+} \tau^{-}$where one of the $\tau$ leptons decays via this background decay chain. No detector simulation is used. Following ref. [55], we require the $K_{L}$ decay position to be in the fiducial volume defined by the radial and longitudinal ranges $10<r<80 \mathrm{~cm}$, $-40<z<120 \mathrm{~cm}$. The background events have an acceptance of $15 \%$ relative to this selection, which is motivated by the long lifetime of the neutralino and by the desire to suppress background from prompt tracks and from material-interaction background.

We form a $\pi^{0}$ candidate from two photons other than $\gamma_{d}^{1} \gamma_{d}^{2}$. Each photon is required to have a lab-frame energy of at least $100 \mathrm{MeV}$. This requirement is tighter than the usual selection in $\tau$ decays [64], and thus leads to a more robust and conservative background estimate. The Belle II crystal calorimeter [46] has only limited ability to determine the flight direction of a photon, and we conservatively ignore this ability altogether. Thus, we take the direction of the photon momentum from its hit position on the surface of the calorimeter, assuming that it originated from the $K_{L}$ decay position, which will be experimentally known from the $\pi^{+} \pi^{-}$decay vertex. Following ref. [64], we require the invariant mass of the $\pi^{0}$ candidate to be within $15 \mathrm{MeV}$ of the known value [62]. Similarly, the invariant mass of the $\eta$ candidate formed from the $\pi^{0}$ candidate and the displaced $\pi^{+} \pi^{-}$pair is required to be within $15 \mathrm{MeV}$ of the known value, corresponding to about 3 times the resolution. Since the $\gamma_{p}^{1} \gamma_{d}^{2}$ and the $\pi^{+} \pi^{-} \gamma^{1} \gamma^{2}$ mass spectra are broad and smooth, the lack of a detector simulation does not significantly impact our rough estimate. We find that these requirements retain $2.5 \%$ of the background events that are within the fiducial region.

We further require that each of the additional photons in the event be unobservable with conservative requirements, namely, escape the detector through the endcap openings in the calorimeter [46] or have a lab-frame energy smaller than $100 \mathrm{MeV}$. This requirement retains $3.9 \%$ of the background events selected up to this point. As a whole, we expect a total of about 300 background events in the Belle II dataset.

As detailed in ref. [55], we further apply the constraints of the signal decay chain to determine the neutrino 4-momentum up to a 2-fold ambiguity. For each of the two solutions, indicated by $i=1,2$, this allows us to compute the calculated neutralino mass $m_{i}$ and the $\tau$ energy $E_{i}$ in the $e^{+} e^{-}$center-of-mass frame. The distributions of these variables are shown in figure 4 for signal events with $m_{\tilde{\chi}_{1}^{0}}=1.1 \mathrm{GeV}$ and for $\tau^{-} \rightarrow \pi^{-} \pi_{p}^{0} K_{L} \nu_{\tau}$ background events. The background plots contain 300 events, illustrating the full Belle II dataset. The $m_{i}$ distributions for signal, which peak at the generated mass, are missing the radiative tails seen in ref. [55], since our signal simulation lacks final-state radiation. The signal $E_{i}$ distributions, which peak at half the center-of-mass energy, show tails that result from the simulated initial-state radiation. The background distributions are very different from those of the signal: events accumulate at high values of $m_{i}$ and away from the correct 

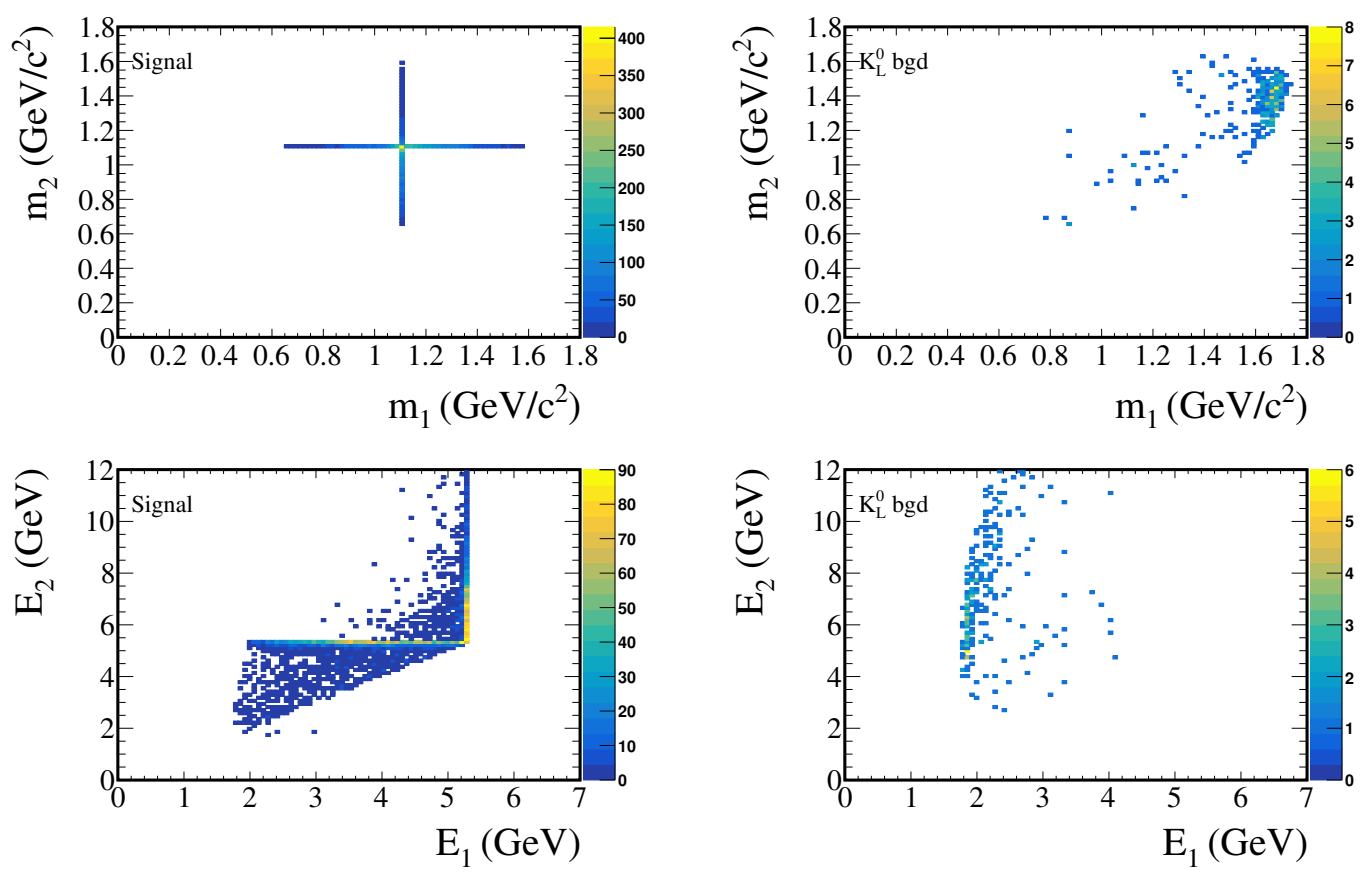

Figure 4. The two solutions for the computed neutralino mass (top plots) and $\tau$ energy (bottom plots) for $\tau^{-} \rightarrow \pi^{-} \tilde{\chi}_{1}^{0}, \tilde{\chi}_{1}^{0} \rightarrow \eta \nu, \eta \rightarrow \pi^{+} \pi^{-} \pi^{0}$ signal events with $m_{\tilde{\chi}_{1}^{0}}=1.1 \mathrm{GeV}$ (left plots) and for $\tau^{-} \rightarrow \pi^{-} \pi_{p}^{0} K_{L} \nu_{\tau}$ background events (right plots).

values of $E_{i}$. Therefore, we conclude that the analysis is essentially background-free, with less background than even in the case of ref. [55].

A related background channel in the $\tilde{\chi}_{1}^{0} \rightarrow \eta \nu_{\tau}$ signal channel is $\tau^{-} \rightarrow \pi^{-} \pi_{p}^{0} K_{L} \nu_{\tau}$ followed by $K_{L} \rightarrow \pi^{ \pm} \ell^{\mp} \nu$. In this case, only the prompt $\pi^{0}$ is available to fake the $\eta$ signal, resulting in a factor of 3 suppression relative to the background from $K_{L} \rightarrow \pi^{+} \pi^{-} \pi^{0}$. This background is further suppressed to the sub-percent level by rejecting DVs formed by a lepton. Overall, its contribution is expected to be smaller than that of $K_{L} \rightarrow \pi^{+} \pi^{-} \pi^{0}$.

Background in the $\tilde{\chi}_{1}^{0} \rightarrow \eta^{\prime} \nu_{\tau}$ channel is much smaller than in the $\tilde{\chi}_{1}^{0} \rightarrow \eta \nu_{\tau}$ channel. This arises from the higher mass of the $\eta^{\prime}$, as well as the fact that the dominant decays $\eta^{\prime} \rightarrow \eta \pi^{+} \pi^{-}$and $\rho \gamma$ provide an additional intermediate hadron ( $\eta$, and to some extent, $\rho$ ) for which a mass cut can be used for background rejection.

In the $\lambda_{312}^{\prime}$ case, there is no $\tau$-decay background that leads to the same final state as the $\tilde{\chi}_{1}^{0} \rightarrow K^{* 0} \nu_{\tau}$ signal. Therefore, the background for this channel is much smaller than our estimate for $\tilde{\chi}_{1}^{0} \rightarrow \eta \nu_{\tau}$. The $\tilde{\chi}_{1}^{0} \rightarrow K_{S} \nu_{\tau}$ channel suffers from $\tau^{-} \rightarrow \pi^{-} K_{S} \nu_{\tau}$ background, which can be suppressed by requiring that the $K_{S}$ momentum vector be inconsistent with the direction between the $\tau^{-}$and $K_{S}$ decay vertices. The neutralino flight length must be long enough for this cut to be effective. Therefore, in section 6 we present results with the $\tilde{\chi}_{1}^{0} \rightarrow K_{S} \nu_{\tau}$ channel only for $c \tau_{\tilde{\chi}_{1}^{0}}>10 \mathrm{~cm}$. 


\section{Sensitivity estimation method}

In what follows we estimate the sensitivity of the Belle II experiment for the $\tau \rightarrow \tilde{\chi}_{1}^{0} M_{1}^{(*)}$, $\tilde{\chi}_{1}^{0} \rightarrow M_{2}^{(*)} \nu_{\tau}$ signal using $e^{-} e^{+} \rightarrow \tau^{+} \tau^{-}$events. First, we consider the visible branching fraction of the neutralino, defined to be the total branching fraction into final states with at least two charged pions, which are necessary for finding the DV. In the $\lambda_{311}^{\prime} \neq 0$ case, we have

$$
\begin{aligned}
\mathcal{B}\left(\tilde{\chi}_{1}^{0} \rightarrow \text { visibles }\right)= & \mathcal{B}\left(\tilde{\chi}_{1}^{0} \rightarrow \nu_{\tau} \pi^{0}\right) \cdot \mathcal{B}\left(\pi^{0} \rightarrow \text { visibles }\right)+\mathcal{B}\left(\tilde{\chi}_{1}^{0} \rightarrow \nu_{\tau} \rho^{0}\right) \cdot \mathcal{B}\left(\rho^{0} \rightarrow \text { visibles }\right) \\
& +\mathcal{B}\left(\tilde{\chi}_{1}^{0} \rightarrow \nu_{\tau} \eta\right) \cdot \mathcal{B}(\eta \rightarrow \text { visibles })+\mathcal{B}\left(\tilde{\chi}_{1}^{0} \rightarrow \nu_{\tau} \eta^{\prime}\right) \cdot \mathcal{B}\left(\eta^{\prime} \rightarrow \text { visibles }\right) \\
& +\mathcal{B}\left(\tilde{\chi}_{1}^{0} \rightarrow \nu_{\tau} \omega\right) \cdot \mathcal{B}(\omega \rightarrow \text { visibles })
\end{aligned}
$$

where the visible decay branching ratios of the mesons are

$$
\begin{aligned}
\mathcal{B}\left(\pi^{0} \rightarrow \text { visibles }\right)= & 0, \\
\mathcal{B}\left(\rho^{0} \rightarrow \text { visibles }\right)= & 1, \\
\mathcal{B}(\omega \rightarrow \text { visibles })= & \mathcal{B}\left(\omega \rightarrow \pi^{+} \pi^{-}\right)+\mathcal{B}\left(\omega \rightarrow \pi^{+} \pi^{-} \pi^{0}\right), \\
\mathcal{B}(\eta \rightarrow \text { visibles })= & \mathcal{B}\left(\eta \rightarrow \pi^{+} \pi^{-} \pi^{0}\right)+\mathcal{B}\left(\eta \rightarrow \pi^{+} \pi^{-} \gamma\right), \\
\mathcal{B}\left(\eta^{\prime} \rightarrow \text { visibles }\right)= & \mathcal{B}\left(\eta^{\prime} \rightarrow \pi^{+} \pi^{-} \eta\right)+\mathcal{B}\left(\eta^{\prime} \rightarrow \rho^{0} \gamma\right) \cdot \mathcal{B}\left(\rho^{0} \rightarrow \text { visibles }\right) \\
& +\mathcal{B}\left(\eta^{\prime} \rightarrow \pi^{0} \pi^{0} \eta\right) \cdot \mathcal{B}(\eta \rightarrow \text { visibles })+\mathcal{B}\left(\eta^{\prime} \rightarrow \omega \gamma\right) \cdot \mathcal{B}(\omega \rightarrow \text { visibles }) .
\end{aligned}
$$

In $\lambda_{312}^{\prime} \neq 0$ scenario, the 'visible' decay branching ratio of the neutralino is

$$
\begin{aligned}
\mathcal{B}\left(\tilde{\chi}_{1}^{0} \rightarrow \text { visibles }\right)= & \mathcal{B}\left(\tilde{\chi}_{1}^{0} \rightarrow \nu_{\tau} K^{* 0}\right) \cdot \mathcal{B}\left(K^{* 0} \rightarrow \text { visibles }\right) \\
& +\mathcal{B}\left(\tilde{\chi}_{1}^{0} \rightarrow \nu_{\tau} K_{S}\right) \cdot \mathcal{B}\left(K_{S} \rightarrow \text { visibles }\right),
\end{aligned}
$$

with

$$
\begin{aligned}
\mathcal{B}\left(K^{* 0} \rightarrow \text { visibles }\right) & =\mathcal{B}\left(K^{* 0} \rightarrow K^{ \pm} \pi^{\mp}\right), \\
\mathcal{B}\left(K_{S} \rightarrow \text { visibles }\right) & =\mathcal{B}\left(K_{S} \rightarrow \pi^{+} \pi^{-}\right) .
\end{aligned}
$$

A summary of the meson branching fractions is given in table 3 .

Next, we consider the signal acceptance and efficiency. Given the similarity between the search proposed here and the one discussed in ref. [55], we use that reference to define the event-selection criteria and their related efficiencies. In principle, a DV can be reconstructed if its position is within an acceptance volume, which we take to be effectively defined by the radial and longitudinal requirements $10<r<80 \mathrm{~cm},-40<z<120 \mathrm{~cm}$. The $r>10$ limit rejects most DVs that arise from material interaction and $K_{S}$ decays. The other requirements ensure a sufficient number of hits for adequate track and vertex reconstruction, given the size of the Belle II tracking systems.

For a DV within the acceptance volume, the efficiency to reconstruct a signal event is $12 \%$, including the impact of reconstructing the two prompt tracks and application of particle-identification criteria on both tracks [55]. Relying on particle-reconstruction efficiencies at the $B$-factories $[69,70]$, we take the efficiency to find any additional $\pi^{0}$ or 
photon that is part of the signal decay to be $70 \%$, and the efficiency for an additional pair of charged pions to be $85 \%$.

While generally, the position of the DV is the decay position of the neutralino, this is not the case when $M_{2}=K_{S}$, because of the significant proper flight distance of the $K_{S}$, $c \tau_{K_{S}}=2.7 \mathrm{~cm}$. As a result, the $M_{2}=K_{S}$ mode is susceptible to background such as $\tau^{-} \rightarrow \pi^{-} K_{S} \nu_{\tau}$. This background will be suppressed by requiring that the $K_{S}$ momentum does not point back to the IP. The efficiency of this requirement is taken to be $90 \%$.

For each value of the neutralino mass $m_{\tilde{\chi}_{1}^{0}}$ and the relevant coupling $\lambda_{31 k}^{\prime}$, we calculate the total number of signal events observed in the experiment,

$$
N_{S}=2 N_{\tau^{-} \tau^{+}} \cdot \mathcal{B}(\tau \rightarrow 1 \text { prong }) \cdot \mathcal{B}\left(\tau \rightarrow \tilde{\chi}_{1}^{0} M_{1}^{(*)}\right) \cdot \mathcal{B}\left(\tilde{\chi}_{1}^{0} \rightarrow \text { visibles }\right) \cdot \epsilon_{\text {acc. }} \cdot \epsilon_{\text {det. }},
$$

where $\mathcal{B}(\tau \rightarrow 1$ prong $) \approx 85 \%$ is the branching fraction for $\tau$ decays into a single track, $M_{1}^{(*)}$ are listed in table $1, \mathcal{B}\left(\tau \rightarrow \tilde{\chi}_{1}^{0} M_{1}^{(*)}\right)$ and $\mathcal{B}\left(\tilde{\chi}_{1}^{0} \rightarrow\right.$ visibles) are calculated as described above, $\epsilon_{\text {acc. }}$ denotes the acceptance, and $\epsilon_{\text {det. }}$ is the reconstruction efficiency discussed above.

The acceptance $\epsilon_{\text {acc. }}$ depends on the neutralino boost, lifetime, and travel direction, as well as the geometry of the acceptance volume. We estimate $\epsilon_{\text {acc. }}$ using a MC simulation with the Pythia $8.243[71,72]$ event generator. We use the Pythia module WeakSingleBoson:ffbar2ffbar (s:gm) to produce $N_{\mathrm{MC}} \sim 1.5 \times 10^{4} e^{-} e^{+} \rightarrow \tau^{-} \tau^{+}$events, including simulation of initial-state radiation. All the simulated $\tau$ leptons undergo the signal decays listed in table 1 exclusively, according to the relative branching ratios computed with the formulas given in section 3 . We estimate $\epsilon_{\text {acc. }}$ with

$$
\epsilon_{\text {acc. }}=\frac{1}{2 N_{\mathrm{MC}}} \sum_{i=1}^{2 N_{\mathrm{MC}}} \epsilon_{\mathrm{acc} .}^{i},
$$

where $\epsilon_{\text {acc. }}^{i}$ is the acceptance for the polar angle $\theta_{i}$ of the $i^{\text {th }}$ simulated neutralino, obtained from

$$
\epsilon_{\text {acc. }}^{i}=e^{-z_{i}^{I} / \lambda_{i}^{z}} \cdot\left(1-e^{-z_{i}^{O} / \lambda_{i}^{z}}\right) .
$$

Here $z_{i}^{I}$ is the $z$ coordinate at which the neutralino enters the acceptance volume, and $z_{i}^{O}$ is the distance traveled inside the acceptance volume:

$$
\begin{aligned}
z_{i}^{I} & \equiv \min \left(Z,\left|R_{I} / \tan \theta_{i}\right|\right), \\
z_{i}^{O} & \equiv \min \left(Z,\left|R_{O} / \tan \theta_{i}\right|\right)-z_{i}^{I},
\end{aligned}
$$

where $R_{I}=10 \mathrm{~cm}, R_{O}=80 \mathrm{~cm}$ are the inner and outer radii of the acceptance volume, $Z=120 \mathrm{~cm}$ for $\tan \theta_{i}>0$ and $40 \mathrm{~cm}$ for $\tan \theta_{i}<0$ are the longitudinal edges of the acceptance volume. The factor

$$
\lambda_{i}^{z} \equiv \beta_{i}^{z} \gamma_{i} c \tau_{\tilde{\chi}_{1}^{0}},
$$

is the average flight distance in the $z$ direction of the neutralino given the lifetime $\tau_{\tilde{\chi}_{1}^{0}}$ and the $z$-direction boost factor $\beta_{i}^{z} \gamma_{i}$ obtained from the Pythia simulation. 

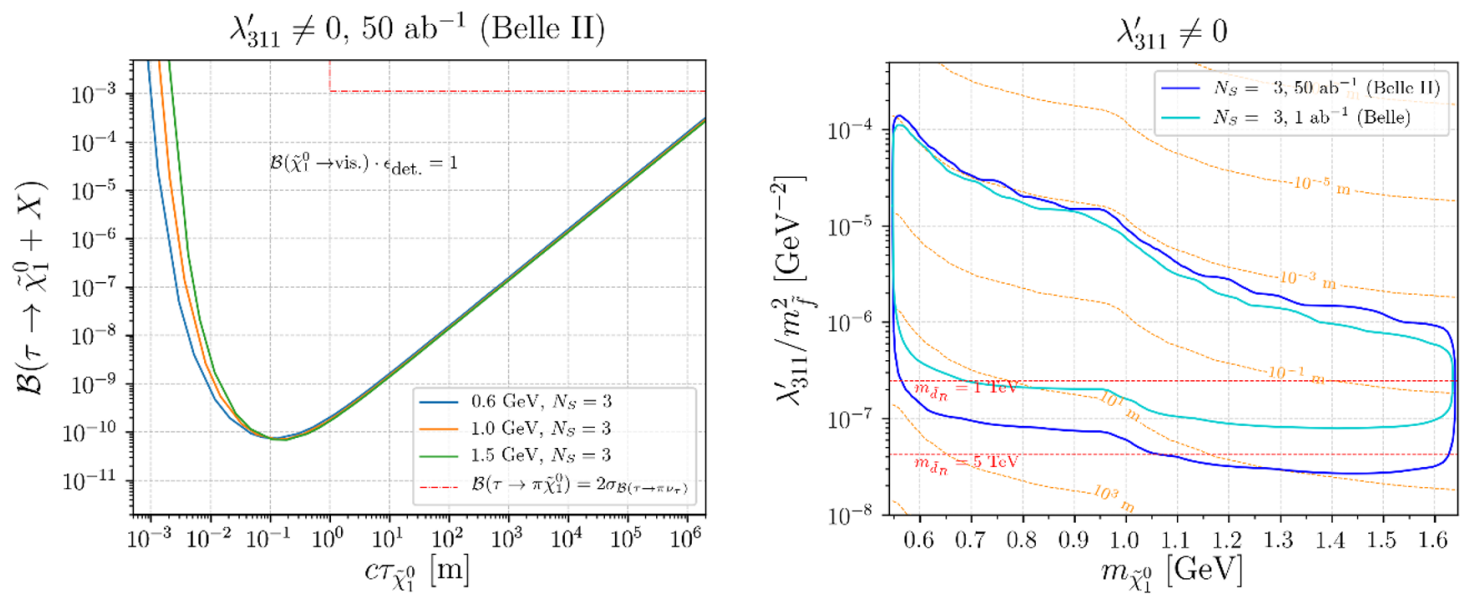

Figure 5. Sensitivity reach for the case $\lambda_{311}^{\prime} \neq 0$. Curves show the parameter values that yield observation of 3 signal events, corresponding to $95 \%$ confidence-level limits for negligible background. Left: model-independent limits on the branching fraction $\mathcal{B}\left(\tau \rightarrow M_{1}^{(*)} \tilde{\chi}_{1}^{0}\right)$, taking $100 \%$ for the branching fraction $\tilde{\chi}_{1}^{0} \rightarrow$ visibles and the detector efficiency $\epsilon_{\text {det. }}$, as a function of $c \tau_{\tilde{\chi}_{1}^{0}}$. Three curves correspond to $m_{\tilde{\chi}_{1}^{0}}=0.6$ (blue), 1.0 (orange), and $1.5 \mathrm{GeV}$ (green). The red dot-dashed line represents the current constraint that arises from the experimental and theoretical uncertainties in $\mathcal{B}\left(\tau^{-} \rightarrow \pi^{-} \nu_{\tau}\right)$, eq. (2.6). This constraint is relevant only for large lifetimes, and is drawn only for $c \tau_{\tilde{\chi}_{1}^{0}}>1 \mathrm{~m}$. Right: model-dependent results on $\lambda_{311}^{\prime} / m_{\tilde{f}}^{2}$ vs. $m_{\tilde{\chi}_{1}^{0}}$ shown for the Belle (light blue, $1 \mathrm{ab}^{-1}$ ) and Belle II (dark blue, $50 \mathrm{ab}^{-1}$ ) data sets. The orange dashed curves show the proper decay length, $c \tau_{\tilde{\chi}_{1}^{0}}$, of the neutralino in intervals of $100 \mathrm{~m}$. The red dashed horizontal lines denote the current limits on " $\lambda_{31 k}^{\prime} / m_{\tilde{q}_{R}}^{2}$ (eq. (2.2)) for squark masses of $1 \mathrm{TeV}$ and $5 \mathrm{TeV}$.

We evaluate $N_{S}$ in a rectangular grid of points in the parameter space $m_{\tilde{\chi}_{1}^{0}}$ vs. $\lambda_{31 k}^{\prime} / m_{\tilde{f}}^{2}$ for $k=1,2$. In the $k=1$ case, we scan $m_{\tilde{\chi}_{1}^{0}}$ in steps of $0.01 \mathrm{GeV}$ for $m_{\tilde{\chi}_{1}^{0}}>0.55 \mathrm{GeV}$, while for $0.547<m_{\tilde{\chi}_{1}^{0}}<0.55 \mathrm{GeV}$ we take the finer steps of $0.001 \mathrm{GeV}$. In the $k=2$ case, we scan $m_{\tilde{\chi}_{1}^{0}}$ in steps of $0.01 \mathrm{GeV}$ for $0.5<m_{\tilde{\chi}_{1}^{0}}<1.28 \mathrm{GeV}$, and in steps of $0.001 \mathrm{GeV}$ in the ranges $0.49<m_{\tilde{\chi}_{1}^{0}}<0.499 \mathrm{GeV}$ and $1.28<m_{\tilde{\chi}_{1}^{0}}<1.3 \mathrm{GeV}$. The ratios $\lambda_{31 k}^{\prime} / m_{\tilde{f}}^{2}$ are scanned between $10^{-10} \mathrm{GeV}^{-2}$ and $8 \times 10^{-4} \mathrm{GeV}^{-2}$, for 49 points in total.

\section{$6 \quad$ Numerical results}

We proceed to discuss the numerical results for both model-independent and modeldependent limits. The model-independent limits are quoted in terms of the branching fraction $\mathcal{B}\left(\tau \rightarrow \tilde{\chi}_{1}^{0} M_{1}^{(*)}\right)$, taking the branching fraction $\mathcal{B}\left(\tilde{\chi}_{1}^{0} \rightarrow\right.$ visibles $)$ and detector efficiency $\epsilon_{\text {det. }}$. to be $100 \%$. These limits are shown as a function of $c \tau_{\tilde{\chi}_{1}^{0}}$, requiring only that the $\tilde{\chi}_{1}^{0}$ decay within the acceptance region. The model-dependent limits are given in terms of the ratio between the RPV coupling and the universal squark mass squared, $\lambda_{31 k}^{\prime} / m_{\tilde{f}}^{2}$, as a function of $m_{\tilde{\chi}_{1}^{0}}$, and use the efficiency estimates described above. Both types of limits are obtained by requiring observation of $N_{S}=3$ signal events at Belle II with a data sample of $50 \mathrm{ab}^{-1}$ for a background expectation of close to zero events, corresponding to $95 \%$ confidence-level limits. The model-dependent limits are presented also for an integrated luminosity of $1 \mathrm{ab}^{-1}$, corresponding to the data already collected by the Belle experiment, 

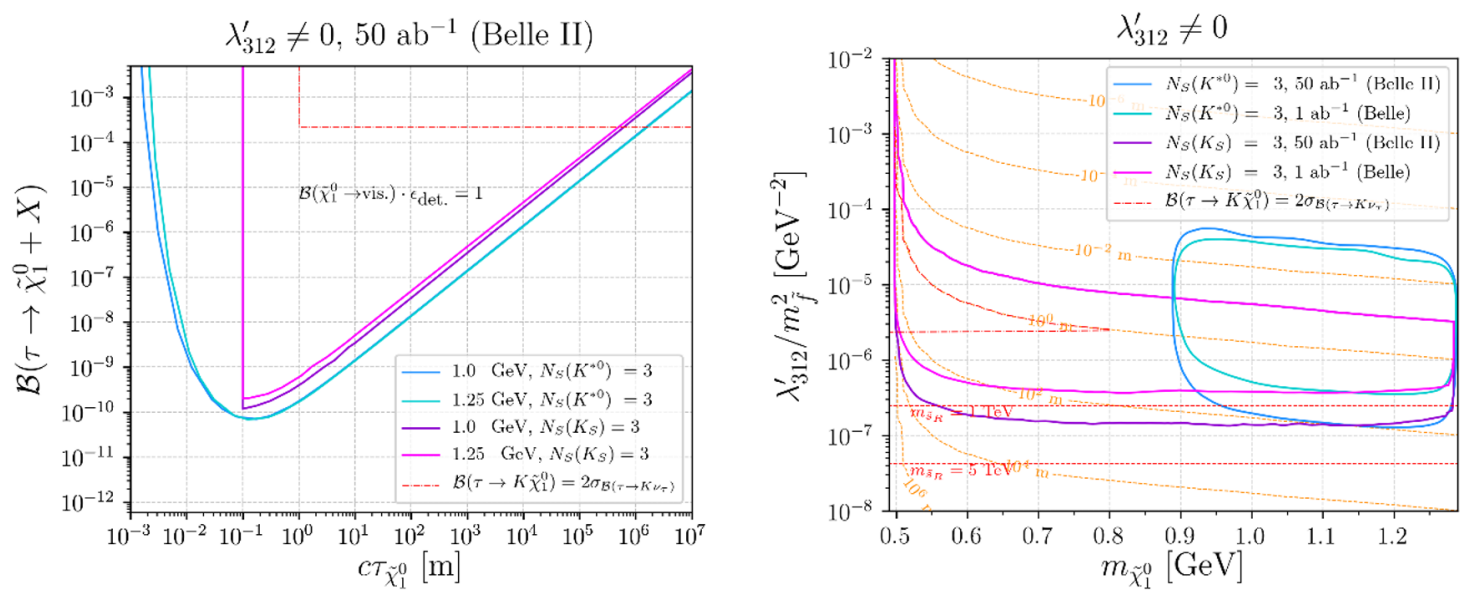

Figure 6. Sensitivity reach for the case $\lambda_{312}^{\prime} \neq 0$. See caption of figure 5 for details. Unlike in figure 5 , the red dot-dashed curve that represents the constraint from $\mathcal{B}\left(\tau^{-} \rightarrow \pi^{-} \nu_{\tau}\right)$ (eq. (2.6)) is relevant also for the model-dependent limits shown in the right panel for $c \tau_{\tilde{\chi}_{1}^{0}}>1 \mathrm{~m}$. The curves for $\tilde{\chi}_{1}^{0} \rightarrow K_{S} \nu_{\tau}$ are shown only for $c \tau_{\tilde{\chi}_{1}^{0}}>10 \mathrm{~cm}$, because of the high background at short flight distances.

and twice the integrated luminosity of BABAR. We note that for small values of $\lambda_{311}^{\prime} / m_{\tilde{f}}^{2}$, the Belle limits are weaker than the Belle II limits by about the $4^{\text {th }}$ root of the ratio of their integrated luminosities, $50^{1 / 4} \sim 2.6$. This is because in the large decay length limit, the number of signal events is proportional to $\left(\lambda_{311}^{\prime} / m_{\tilde{f}}^{2}\right)^{4}$.

In figure 5 we show the limits for $\lambda_{311}^{\prime} \neq 0$. The model-independent limits are given in the left panel for three neutralino mass values: $0.6 \mathrm{GeV}$ (blue), $1.0 \mathrm{GeV}$ (orange), and $1.5 \mathrm{GeV}$ (green) using the full Belle II data. Also plotted is the limit extracted from the current uncertainties in the measured branching fraction for $\tau^{-} \rightarrow \pi^{-} \nu_{\tau}$ and its theoretical prediction (red dot-dashed lines), eq. (2.6). We find that Belle II may probe $\mathcal{B}\left(\tau \rightarrow \tilde{\chi}_{1}^{0}+X\right)$ values many orders of magnitude smaller than the present experimental upper bound, for $m_{\tilde{\chi}_{1}^{0}}$ below $m_{\tau}$, with the best sensitivity obtained for $c \tau_{\tilde{\chi}_{1}^{0}} \sim 10-20 \mathrm{~cm}$.

The right panel of figure 5 displays the model-dependent limits on $\lambda_{31 k}^{\prime} / m_{\tilde{f}}^{2}$ that we expect with the full Belle and Belle II data sets. Also shown is the upper bound of eq. (2.2) on the ratio $\lambda_{311}^{\prime} / m_{\tilde{q}_{R}}^{2}$ for the benchmark squark masses of 1 and $5 \mathrm{TeV}$. Since the limit of eq. (2.2) has a different power of $m_{\tilde{f}}$, its comparison to our results depends on the assumed value for $m_{\tilde{f}}$. The upper bound derived from the uncertainty on $\tau^{-} \rightarrow \pi^{-} \tilde{\chi}_{1}^{0}$ is irrelevant here, as it corresponds to values of $\lambda_{311}^{\prime} / m_{\tilde{f}}^{2}$ and $m_{\tilde{\chi}_{1}^{0}}$ where $c \tau_{\tilde{\chi}_{1}^{0}}<1 \mathrm{~m}$, in which this bound does not apply. We find that the search we propose is more sensitive than the limit of eq. (2.2) for sfermion masses of up to about $5 \mathrm{TeV}$. The right plot of figure 5 also shows orange dashed isocurves of the proper decay length $c \tau_{\tilde{\chi}_{1}^{0}}$ of the neutralino in the shown parameter space.

Figure 6 gives the results for the $\lambda_{312}^{\prime} \neq 0$ scenario, treating separately the $M_{2}=K_{S}$ and $M_{2}^{*}=K^{* 0}$ cases. We overlap the limits derived from $\tau \rightarrow K \nu_{\tau}$ for $c \tau \gtrsim 1 \mathrm{~m}$ only. As mentioned above, the $K_{S}$ channel suffers from large background at short distances. We incorporate this by showing the limits expected for the $K_{S}$ channel only for $c \tau_{\tilde{\chi}_{1}^{0}}>10 \mathrm{~cm}$ 
(magenta and purple curves in the two panels). Despite this shortcoming of the $K_{S}$ channel, its advantage is that it is sensitive to $m_{\tilde{\chi}_{1}^{0}}<0.9 \mathrm{GeV}$. Overall, comparison of figures 5 and 6 shows that the scenario $\lambda_{311}^{\prime} \neq 0$ is probed with greater sensitivity, by almost an order of magnitude, than the scenario $\lambda_{312}^{\prime} \neq 0$.

\section{Conclusions}

In recent years, searches for long-lived particles (LLPs) have become an important means for probing new physics, particularly at the LHC. A well-motivated scenario for new physics is R-parity-violating (RPV) supersymmetry with light, long-lived neutralinos. Such neutralinos may be produced at colliders and lead to an exotic displaced-vertex signature. In this work, we develop a method for studying the scenario in which the neutralino mass $m_{\tilde{\chi}_{1}^{0}}$ is roughly between $0.5 \mathrm{GeV}$ and $m_{\tau}-m_{\pi}$. We study two benchmark scenarios in which one of the RPV operators, $\lambda_{311}^{\prime} L_{3} Q_{1} \bar{D}_{1}$ or $\lambda_{312}^{\prime} L_{3} Q_{1} \bar{D}_{2}$, is non-vanishing. Each of these operators induces $\tau$ decay into the neutralino plus an accompanying meson $M_{1}^{(*)}$, and also the neutralino decay into a meson $M_{2}^{(*)}$ plus a tau neutrino. We propose to conduct the search at electron-positron $B$-factories, which are particularly suitable for the study of rare $\tau$ decays. Specifically, the BABAR and Belle experiments have collected a combined data sample of $1.4 \times 10^{9} e^{+} e^{-} \rightarrow \tau^{+} \tau^{-}$events, and the ongoing Belle II experiment is scheduled to collect $4.6 \times 10^{10}$ events in the coming years.

For both RPV operators, we consider in detail the possible decays of the $\tau$ lepton and of the neutralino, defining the final states and displaced-vertex signatures that we propose to study. Correspondingly, we perform Monte-Carlo simulations to determine the leading background and the signal events acceptance. In particular, simulation is also used to estimate the largest source of background, $\tau^{-} \rightarrow \pi^{-} K_{L} \nu_{\tau}$ with $K_{L} \rightarrow \pi^{+} \pi^{-} \pi^{0}$, which impacts the $\tilde{\chi}_{1}^{0} \rightarrow \eta \nu_{\tau}, \eta \rightarrow \pi^{+} \pi^{-} \pi^{0}$ signal channel. After using the constraints of the signal decay to obtain the neutralino mass and the $\tau$ energy, we expect the effective background yield in this channel to be negligible even in the full Belle II sample. In the other major channels, the expected background is even smaller.

Using our estimated acceptance, efficiency, and estimated negligible background yield, we present numerical results for both model-independent and model-dependent limits. In both scenarios, the model-independent limits on $\mathcal{B}\left(\tau \rightarrow X \tilde{\chi}_{1}^{0}\right)$ are orders of magnitude tighter than the current bounds stemming from the combined experimental and theoretical uncertainties in the branching ratio for $\tau$ decay into a charged hadron plus a neutrino. The model-dependent limits on $\lambda_{311}^{\prime} / m_{\tilde{f}}^{2}$ are up to two orders of magnitude tighter than the limits obtained by recasting [59] the $p p \rightarrow W^{\prime} \rightarrow \tau \nu_{\tau}$ search for squark mass $m_{\tilde{q}_{R}}=1 \mathrm{TeV}$, and comparable for $m_{\tilde{q}_{R}}=5 \mathrm{TeV}$. The limits on $\lambda_{312}^{\prime} / m_{\tilde{f}}^{2}$ are comparable with the $p p \rightarrow$ $W^{\prime} \rightarrow \tau \nu_{\tau}$ for $m_{\tilde{f}}=1 \mathrm{TeV}$. We note that the bounds obtained from the $p p \rightarrow W^{\prime} \rightarrow \tau \nu_{\tau}$ search are relevant only when the RPV process involves a virtual squark, while our results also probe the case of a virtual stau and sneutrino.

Together with ref. [55], this work exemplifies the usefulness of $B$-factory experiments for searching for new physics related to the $\tau$ lepton, e.g., long-lived particles produced in $\tau$ decays. We propose that further scenarios be pursued along this direction. 


\begin{tabular}{|lcc|}
\hline Constant & Value $[\mathrm{MeV}]$ & Ref. \\
\hline$f_{\pi^{ \pm}}$ & 130.2 & {$[62]$} \\
$f_{\rho}$ & 209 & {$[76]$} \\
$f_{a_{1}}$ & 209 & {$[74,75]$} \\
$f_{K^{ \pm}}$ & 155.7 & {$[62]$} \\
$f_{K^{*}}$ & 230 & {$[77]$} \\
$f_{\omega}$ & 195.3 & {$[76]$} \\
$f_{\eta}^{\bar{d} d}$ & 49.42 & {$[73]$} \\
$f_{\eta^{\prime}}^{\bar{d} d}$ & 111.43 & {$[73]$} \\
\hline
\end{tabular}

Table 2. Values of the decay constants used in this work.

\begin{tabular}{|c|c|}
\hline Decay & Branching fraction \\
\hline$\omega \rightarrow \pi^{+} \pi^{-} \pi^{0}$ & 0.893 \\
$\omega \rightarrow \pi^{+} \pi^{-}$ & 0.0153 \\
$\eta \rightarrow \pi^{+} \pi^{-} \pi^{0}$ & 0.2292 \\
$\eta \rightarrow \pi^{+} \pi^{-} \gamma$ & 0.0422 \\
$\eta \rightarrow \gamma \gamma$ & 0.3941 \\
$\eta \rightarrow \pi^{0} \pi^{0} \pi^{0}$ & 0.3268 \\
$\eta^{\prime} \rightarrow \pi^{+} \pi^{-} \eta$ & 0.426 \\
$\eta^{\prime} \rightarrow \rho \gamma$ & 0.289 \\
$\eta^{\prime} \rightarrow \pi^{0} \pi^{0} \eta$ & 0.228 \\
$\eta^{\prime} \rightarrow \omega \gamma$ & 0.0262 \\
\hline$K_{S} \rightarrow \pi^{+} \pi^{-}$ & 0.692 \\
$K^{* 0} \rightarrow K^{ \pm} \pi^{\mp}$ & 0.665 \\
\hline
\end{tabular}

Table 3. Branching fractions for the meson decays considered in our analysis. The values are reproduced from ref. [62].

\section{A Meson decay constants and branching fractions}

The decay constant for the pseudoscalar current is [38]

$$
f_{M}^{S} \equiv\left\langle 0\left|\bar{q}_{1} \gamma^{5} q_{2}\right| M\right\rangle=i \frac{m_{M}^{2}}{m_{q_{1}}+m_{q_{2}}} f_{M},
$$

where $M$ is the meson mass and $q_{1}$ and $q_{2}$ its valence quarks. The values of $f_{M}$ used in section 3 are summarized in table 2 . Because the tensor decay constants $f_{M^{*}}^{T}$ are not known for all mesons, following ref. [38], we assume $f_{M^{*}}^{T} \approx f_{M}$. Note that for the neutral $\pi$ and $\rho$ mesons, $f_{\pi^{0}, \rho^{0}}=f_{\pi, \rho} / \sqrt{2}$ in eq. A.1. For the $\eta$ and $\eta^{\prime}$ mesons, the decay constants are obtained from ref. [73], which takes into account the mixing between the $\eta^{0}$ and $\eta^{8} \mathrm{SU}(3)$ flavor states. Distinct calculations of the $a_{1}(1260)$ meson decay constant find different values around the $\rho$ decay constant $[74,75]$. For definiteness, we chose $f_{a_{1}}=f_{\rho}$.

A summary of the relevant meson branching fractions is given in table 3 . 


\section{Acknowledgments}

We would like to thank Jordy de Vries, Marcela González, Sergey Kovalenko, and Torbjörn Sjöstrand for useful discussions. Z.S.W. is supported partly by the Ministry of Science and Technology (MoST) of Taiwan with grant number MoST-109-2811-M-007-509, and partly by the Ministry of Science, ICT \& Future Planning of Korea, the Pohang City Government, and the Gyeongsangbuk-do Provincial Government through the Young Scientist Training Asia Pacific Economic Cooperation program of the Asia Pacific Center for Theoretical Physics. C.O.D. acknowledges support from FONDECYT (Chile) Grant No. 1170171 and ANID (Chile) PIA/APOYO AFB 180002. J.C.H. acknowledges support from grant FONDECYT (Chile) No. 1201673. N.A.N. was supported by ANID (Chile) under the grant ANID REC Convocatoria Nacional Subvención a Instalación en la Academia Convocatoria Año 2020, PAI77200092.

Open Access. This article is distributed under the terms of the Creative Commons Attribution License (CC-BY 4.0), which permits any use, distribution and reproduction in any medium, provided the original author(s) and source are credited.

\section{References}

[1] H.P. Nilles, Supersymmetry, supergravity and particle physics, Phys. Rept. 110 (1984) 1 [INSPIRE].

[2] S.P. Martin, A supersymmetry primer, Adv. Ser. Direct. High Energy Phys. 21 (2010) 1 [hep-ph/9709356] [INSPIRE].

[3] E. Gildener, Gauge symmetry hierarchies, Phys. Rev. D 14 (1976) 1667 [INSPIRE].

[4] M.J.G. Veltman, The infrared- ultraviolet connection, Acta Phys. Polon. B 12 (1981) 437 [INSPIRE].

[5] H.K. Dreiner, An introduction to explicit R-parity violation, Adv. Ser. Direct. High Energy Phys. 21 (2010) 565 [hep-ph/9707435] [INSPIRE].

[6] R. Barbier et al., R-parity violating supersymmetry, Phys. Rept. 420 (2005) 1 [hep-ph/0406039] [INSPIRE].

[7] R.N. Mohapatra, Supersymmetry and R-parity: an overview, Phys. Scripta 90 (2015) 088004 [arXiv: 1503.06478] [INSPIRE].

[8] L.E. Ibáñez and G.G. Ross, Discrete gauge symmetries and the origin of baryon and lepton number conservation in supersymmetric versions of the standard model, Nucl. Phys. B $\mathbf{3 6 8}$ (1992) 3 [INSPIRE].

[9] H.K. Dreiner, M. Hanussek and C. Luhn, What is the discrete gauge symmetry of the R-parity violating MSSM?, Phys. Rev. D 86 (2012) 055012 [arXiv:1206.6305] [INSPIRE].

[10] ATLAS collaboration, Search for photonic signatures of gauge-mediated supersymmetry in $13 \mathrm{TeV}$ pp collisions with the ATLAS detector, Phys. Rev. D 97 (2018) 092006 [arXiv: 1802.03158] [INSPIRE].

[11] CMS collaboration, Search for gauge-mediated supersymmetry in events with at least one photon and missing transverse momentum in pp collisions at $\sqrt{s}=13 \mathrm{TeV}$, Phys. Lett. B 780 (2018) 118 [arXiv:1711.08008] [INSPIRE]. 
[12] CMS collaboration, Search for supersymmetry in final states with photons and missing transverse momentum in proton-proton collisions at 13 TeV, JHEP 06 (2019) 143 [arXiv: 1903.07070] [INSPIRE].

[13] CMS collaboration, Search for supersymmetry in proton-proton collisions at 13 TeV in final states with jets and missing transverse momentum, JHEP 10 (2019) 244 [arXiv: 1908.04722] [INSPIRE].

[14] ATLAS collaboration, Search for new phenomena in final states with large jet multiplicities and missing transverse momentum using $\sqrt{s}=13$ TeV proton-proton collisions recorded by ATLAS in Run 2 of the LHC, JHEP 10 (2020) 062 [arXiv:2008.06032] [InSPIRE].

[15] J. Alimena et al., Searching for long-lived particles beyond the Standard Model at the Large Hadron Collider, J. Phys. G 47 (2020) 090501 [arXiv: 1903.04497] [InSPIRE].

[16] L. Lee, C. Ohm, A. Soffer and T.-T. Yu, Collider searches for long-lived particles beyond the standard model, Prog. Part. Nucl. Phys. 106 (2019) 210 [arXiv:1810.12602] [InSPIRE].

[17] D. Curtin et al., Long-lived particles at the energy frontier: the mathusla physics Case, Rept. Prog. Phys. 82 (2019) 116201 [arXiv: 1806.07396] [INSPIRE].

[18] D. Choudhury and S. Sarkar, A supersymmetric resolution of the KARMEN anomaly, Phys. Lett. $B 374$ (1996) 87 [hep-ph/9511357] [INSPIRE].

[19] D. Choudhury, H.K. Dreiner, P. Richardson and S. Sarkar, A supersymmetric solution to the KARMEN time anomaly, Phys. Rev. D 61 (2000) 095009 [hep-ph/9911365] [InSPIRE].

[20] G. Bélanger, F. Boudjema, A. Pukhov and S. Rosier-Lees, A lower limit on the neutralino mass in the MSSM with nonuniversal gaugino masses, in the proceedings of the $10^{\text {th }}$ International Conference on Supersymmetry and Unification of Fundamental Interactions (SUSY02), June 17-23, Hamburg, Germany (2002), hep-ph/0212227 [INSPIRE].

[21] A. Bottino, N. Fornengo and S. Scopel, Light relic neutralinos, Phys. Rev. D 67 (2003) 063519 [hep-ph/0212379] [INSPIRE].

[22] G. Bélanger, F. Boudjema, A. Cottrant, A. Pukhov and S. Rosier-Lees, Lower limit on the neutralino mass in the general MSSM, JHEP 03 (2004) 012 [hep-ph/0310037] [INSPIRE].

[23] D. Albornoz Vasquez, G. Bélanger, C. Boehm, A. Pukhov and J. Silk, Can neutralinos in the MSSM and NMSSM scenarios still be light?, Phys. Rev. D 82 (2010) 115027 [arXiv: 1009.4380] [INSPIRE].

[24] L. Calibbi, J.M. Lindert, T. Ota and Y. Takanishi, Cornering light neutralino dark matter at the LHC, JHEP 10 (2013) 132 [arXiv:1307.4119] [INSPIRE].

[25] I. Gogoladze, J.D. Lykken, C. Macesanu and S. Nandi, Implications of a massless neutralino for neutrino physics, Phys. Rev. D 68 (2003) 073004 [hep-ph/0211391] [INSPIRE].

[26] H.K. Dreiner, S. Heinemeyer, O. Kittel, U. Langenfeld, A.M. Weber and G. Weiglein, Mass bounds on a very light neutralino, Eur. Phys. J. C 62 (2009) 547 [arXiv:0901.3485] [INSPIRE].

[27] J.A. Grifols, E. Masso and S. Peris, Photinos from gravitational collapse, Phys. Lett. B 220 (1989) 591 [INSPIRE].

[28] J.R. Ellis, K.A. Olive, S. Sarkar and D.W. Sciama, Low mass photinos and supernova SN1987A, Phys. Lett. B 215 (1988) 404 [INSPIRE]. 
[29] K. Lau, Constraints on supersymmetry from SN1987A, Phys. Rev. D 47 (1993) 1087 [INSPIRE].

[30] H.K. Dreiner, C. Hanhart, U. Langenfeld and D.R. Phillips, Supernovae and light neutralinos: SN1987A bounds on supersymmetry revisited, Phys. Rev. D 68 (2003) 055004 [hep-ph/0304289] [INSPIRE].

[31] H.K. Dreiner, J.-F. Fortin, J. Isern and L. Ubaldi, White dwarfs constrain dark forces, Phys. Rev. D 88 (2013) 043517 [arXiv: 1303.7232] [InSPIRE].

[32] S. Profumo, Hunting the lightest lightest neutralinos, Phys. Rev. D 78 (2008) 023507 [arXiv:0806.2150] [INSPIRE].

[33] H.K. Dreiner, M. Hanussek, J.S. Kim and S. Sarkar, Gravitino cosmology with a very light neutralino, Phys. Rev. D 85 (2012) 065027 [arXiv:1111.5715] [InSPIRE].

[34] D. Hooper and T. Plehn, Supersymmetric dark matter: how light can the LSP be?, Phys. Lett. B 562 (2003) 18 [hep-ph/0212226] [INSPIRE].

[35] A. Bottino, N. Fornengo and S. Scopel, Phenomenology of light neutralinos in view of recent results at the CERN Large Hadron Collider, Phys. Rev. D 85 (2012) 095013 [arXiv:1112.5666] [INSPIRE].

[36] G. Bélanger, G. Drieu La Rochelle, B. Dumont, R.M. Godbole, S. Kraml and S. Kulkarni, LHC constraints on light neutralino dark matter in the MSSM, Phys. Lett. B $\mathbf{7 2 6}$ (2013) 773 [arXiv: 1308.3735] [INSPIRE].

[37] P. Bechtle et al., Killing the CMSSM softly, Eur. Phys. J. C 76 (2016) 96 [arXiv: 1508.05951] [INSPIRE].

[38] J. de Vries, H.K. Dreiner and D. Schmeier, R-parity violation and light neutralinos at SHiP and the LHC, Phys. Rev. D 94 (2016) 035006 [arXiv:1511.07436] [INSPIRE].

[39] Z.S. Wang and K. Wang, Long-lived light neutralinos at future Z-factories, Phys. Rev. D 101 (2020) 115018 [arXiv: 1904.10661] [INSPIRE].

[40] Z.S. Wang and K. Wang, Physics with far detectors at future lepton colliders, Phys. Rev. D 101 (2020) 075046 [arXiv:1911.06576] [INSPIRE].

[41] J.C. Helo, M. Hirsch and Z.S. Wang, Heavy neutral fermions at the high-luminosity LHC, JHEP 07 (2018) 056 [arXiv:1803.02212] [INSPIRE].

[42] D. Dercks, J. De Vries, H.K. Dreiner and Z.S. Wang, R-parity violation and light neutralinos at CODEX-b, FASER, and MATHUSLA, Phys. Rev. D 99 (2019) 055039 [arXiv: 1810.03617] [INSPIRE].

[43] D. Dercks, H.K. Dreiner, M. Hirsch and Z.S. Wang, Long-lived fermions at AL3X, Phys. Rev. D 99 (2019) 055020 [arXiv: 1811.01995] [InSPIRE].

[44] H.K. Dreiner, J.Y. Günther and Z.S. Wang, R-parity violation and light neutralinos at ANUBIS and MAPP, arXiv:2008.07539 [INSPIRE].

[45] BES-III collaboration, Tau mass measurement at BES-III, EPJ Web Conf. 212 (2019) 08005 [INSPIRE].

[46] BELLE-II collaboration, Belle II technical design report, arXiv:1011.0352 [INSPIRE].

[47] BeLle-II collaboration, The Belle II physics book, PTEP 2019 (2019) 123 C01 [Erratum ibid. 2020 (2020) 029201] [arXiv: 1808.10567] [INSPIRE]. 
[48] BaBAR collaboration, The BaBar detector, Nucl. Instrum. Meth. A 479 (2002) 1 [hep-ex/0105044] [INSPIRE].

[49] A. Abashian et al., The Belle detector, Nucl. Instrum. Meth. A 479 (2002) 117 [InSPIRE].

[50] B. Batell, M. Pospelov and A. Ritz, Probing a secluded U(1) at B-factories, Phys. Rev. D 79 (2009) 115008 [arXiv:0903.0363] [inSPIRE].

[51] L. Canetti, M. Drewes and B. Garbrecht, Probing leptogenesis with GeV-scale sterile neutrinos at LHCb and Belle II, Phys. Rev. D 90 (2014) 125005 [arXiv:1404.7114] [INSPIRE].

[52] M.J. Dolan, T. Ferber, C. Hearty, F. Kahlhoefer and K. Schmidt-Hoberg, Revised constraints and Belle II sensitivity for visible and invisible axion-like particles, JHEP 12 (2017) 094 [arXiv: 1709.00009] [INSPIRE].

[53] M.K. Sullivan and D. Fryberger, Magnetic charge search for the BELLE II detector, arXiv: 1707.05295 [INSPIRE].

[54] G. Cvetič and C.S. Kim, Sensitivity limits on heavy-light mixing $\left|U_{\mu N}\right|^{2}$ from lepton number violating B meson decays, Phys. Rev. D 96 (2017) 035025 [Erratum ibid. 102 (2020) 019903] [Erratum ibid. 102 (2020) 039902] [arXiv:1705.09403] [INSPIRE].

[55] C.O. Dib, J.C. Helo, M. Nayak, N.A. Neill, A. Soffer and J. Zamora-Saa, Searching for a sterile neutrino that mixes predominantly with $\nu_{\tau}$ at B factories, Phys. Rev. D 101 (2020) 093003 [arXiv: 1908.09719] [INSPIRE].

[56] A. Filimonova, R. Schäfer and S. Westhoff, Probing dark sectors with long-lived particles at BELLE II, Phys. Rev. D 101 (2020) 095006 [arXiv:1911.03490] [InSPIRE].

[57] M. Duerr, T. Ferber, C. Hearty, F. Kahlhoefer, K. Schmidt-Hoberg and P. Tunney, Invisible and displaced dark matter signatures at Belle II, JHEP 02 (2020) 039 [arXiv:1911.03176] [INSPIRE].

[58] SHIP collaboration, The experimental facility for the Search for Hidden Particles at the CERN SPS, 2019 JINST 14 P03025 [arXiv:1810.06880] [INSPIRE].

[59] S. Bansal, A. Delgado, C. Kolda and M. Quirós, Constraining R-parity-violating couplings in $\tau$-processes at the LHC and in electroweak precision measurements, Phys. Rev. D 100 (2019) 093005 [arXiv: 1906.01063] [INSPIRE].

[60] ATLAS collaboration, Search for high-mass resonances decaying to $\tau \nu$ in pp collisions at $\sqrt{s}=13 \mathrm{TeV}$ with the ATLAS detector, Phys. Rev. Lett. 120 (2018) 161802 [arXiv: 1801.06992] [INSPIRE].

[61] CMS collaboration, Search for a $W^{\prime}$ boson decaying to a $\tau$ lepton and a neutrino in proton-proton collisions at $\sqrt{s}=13 \mathrm{TeV}$, Phys. Lett. B 792 (2019) 107 [arXiv:1807.11421] [INSPIRE].

[62] Particle Data Group collaboration, Review of particle physics, PTEP 2020 (2020) $083 \mathrm{C} 01$

[63] R. Decker and M. Finkemeier, Radiative corrections to the decay $\tau \rightarrow \pi \tau_{\nu}$, Nucl. Phys. B Proc. Suppl. 40 (1995) 453 [hep-ph/9411316] [INSPIRE].

[64] BABAR collaboration, Studies of $\tau^{-} \rightarrow \eta K_{\nu}$ and $\tau^{-} \rightarrow \eta \pi^{-} \nu_{\tau}$ at BaBar and a search for a second-class current, Phys. Rev. D 83 (2011) 032002 [arXiv: 1011.3917] [InSPIRE]. 
[65] A. Pich, Precision tau physics, Prog. Part. Nucl. Phys. 75 (2014) 41 [arXiv:1310.7922] [INSPIRE].

[66] S. Nussinov and A. Soffer, Estimate of the branching fraction of $\tau \rightarrow \pi \eta^{\prime} \nu_{\tau}$, Phys. Rev. D 80 (2009) 033010 [arXiv:0907.3628] [INSPIRE].

[67] S. Nussinov and A. Soffer, Estimate of the branching fraction $\tau \rightarrow \eta \pi^{-} \nu_{\tau}$, the $a(0)-(980)$, and non-standard weak interactions, Phys. Rev. D 78 (2008) 033006 [arXiv:0806.3922] [INSPIRE].

[68] D.J. Lange, The EvtGen particle decay simulation package, Nucl. Instrum. Meth. A 462 (2001) 152 [INSPIRE].

[69] T. Allmendinger et al., Track finding efficiency in BaBar, Nucl. Instrum. Meth. A $\mathbf{7 0 4}$ (2013) 44 [arXiv: 1207. 2849] [INSPIRE].

[70] BABAR collaboration, The BABAR detector: upgrades, operation and performance, Nucl. Instrum. Meth. A $\mathbf{7 2 9}$ (2013) 615 [arXiv:1305.3560] [INSPIRE].

[71] T. Sjöstrand, S. Mrenna and P.Z. Skands, PYTHIA 6.4 physics and manual, JHEP 05 (2006) 026 [hep-ph/0603175] [INSPIRE].

[72] T. Sjöstrand, S. Mrenna and P.Z. Skands, A brief introduction to PYTHIA 8.1, Comput. Phys. Commun. 178 (2008) 852 [arXiv:0710.3820] [INSPIRE].

[73] H.K. Dreiner, S. Grab, D. Koschade, M. Krämer, B. O'Leary and U. Langenfeld, Rare meson decays into very light neutralinos, Phys. Rev. D 80 (2009) 035018 [arXiv:0905.2051] [INSPIRE].

[74] J.C.R. Bloch, Y.L. Kalinovsky, C.D. Roberts and S.M. Schmidt, Describing a(1) and b(1) decays, Phys. Rev. D 60 (1999) 111502 [nucl-th/9906038] [InSPIRE].

[75] H.-Y. Cheng, Hadronic charmed meson decays involving axial vector mesons, Phys. Rev. D 67 (2003) 094007 [hep-ph/0301198] [INSPIRE].

[76] D. Ebert, R.N. Faustov and V.O. Galkin, Relativistic treatment of the decay constants of light and heavy mesons, Phys. Lett. B 635 (2006) 93 [hep-ph/0602110] [INSPIRE].

[77] H.K. Dreiner, M. Krämer and B. O'Leary, Bounds on R-parity violating supersymmetric couplings from leptonic and semi-leptonic meson decays, Phys. Rev. D 75 (2007) 114016 [hep-ph/0612278] [INSPIRE]. 